\title{
Stress relaxation in epithelial monolayers is controlled by the actomyosin cortex
}

Nargess Khalilgharibi ${ }^{1,2}$, Jonathan Fouchard ${ }^{1}$, Nina Asadipour ${ }^{3}$, Ricardo Barrientos ${ }^{2,4}$, Maria Duda ${ }^{4}$, Alessandra Bonfanti ${ }^{5}$, Amina Yonis ${ }^{1,6}$, Andrew Harris ${ }^{1,7,8,9}$, Payman Mosaffa $^{3}$, Yasuyuki Fujita ${ }^{10}$, Alexandre Kabla ${ }^{5}$, Yanlan $\mathrm{MaO}^{4}$, Buzz Baum ${ }^{4,11}$, José J Muñoz ${ }^{3,12}$, Mark Miodownik ${ }^{13}$, Guillaume Charras ${ }^{1,6,11}$

(1) London Centre for Nanotechnology, University College London, Gower Street, London WC1E 6BT, UK

(2) Centre for Computation, Mathematics and Physics in the Life Sciences and Experimental Biology (CoMPLEX), University College London, Gower Street, London WC1E 6BT, UK

(3) Laboratori de Càlcul Numèric (LaCàN), Dept. Mathematics, Esc. d'Enginyeria Barcelona Est (EEBE), Universitat Politècnica de Catalunya - Barcelona Tech (UPC), 08036, Barcelona, Spain

(4) MRC Laboratory for Molecular Cell Biology, University College London, Gower Street, London WC1E 6BT, United Kingdom

(5) Department of Mechanical Engineering, Cambridge University, UK

(6) Department of Cell and Developmental Biology, University College London, UK

(7) Department of Physics, University College London, London WC1E 6BT, UK

(8) Engineering Doctorate Program, Department of Chemistry, University College London, London WC1H OAJ, UK

(9) Department of Bioengineering and Biophysics Program, University of California, Berkeley, 648 Stanley Hall MC 1762, Berkeley, CA 94720, USA

(10) Institute for Genetic Medicine, Hokkaido University, Japan

(11) Institute for the Physics of Living Systems, University College London, UK

(12) Barcelona Graduate School of Mathematics (BGSMath), Spain

(13) Department of Mechanical Engineering, University College London, London WC1E 7JE, UK

*Corresponding author: g.charras@ucl.ac.uk 


\section{Abstract}

Epithelial monolayers are one-cell thick tissue sheets that separate internal and external environments. As part of their function, they have to withstand extrinsic mechanical stresses applied at high strain rates. However, little is known about how monolayers respond to mechanical deformations. Here, by subjecting suspended epithelial monolayers to stretch, we find that they dissipate stresses on a minute time-scale in a process that involves an increase in monolayer length, pointing to active remodelling of cell architecture during relaxation. Strikingly, monolayers consisting of tens of thousands of cells relax stress with similar dynamics to single rounded cells and both respond similarly to perturbations of actomyosin. By contrast, cell-cell junctional complexes and intermediate filaments do not relax tissue stress, but form stable connections between cells, allowing monolayers to behave rheologically as single cells. Taken together our data show that actomyosin dynamics governs the rheological properties of epithelial monolayers, dissipating applied stresses, and enabling changes in monolayer length.

\section{Introduction}

Epithelial monolayers line most of the surfaces and internal cavities of the body. They act as physical barriers that subdivide the internal environment into discrete compartments and separate it from the external environment. To fulfil this role, epithelia must withstand substantial mechanical stresses ${ }^{1-4}$. During development, strain in epithelia evolves slowly with strain rates of $\sim 0.04 \% . \mathrm{s}^{-1}$ as a result of forces generated elsewhere in the embryo ${ }^{5}$; while in adult animals, strain rates of $10-100 \% . \mathrm{s}^{-1}$ are observed during the normal function of organs and tissues ${ }^{6-11}$. While in some organs, such as the lung, epithelia are subjected to deformations lasting only seconds, in others such as the skin, the intestine, or the bladder, large deformations can be sustained for durations of minutes ${ }^{11-13}$. In addition, organisms need to withstand external mechanical insults. Thus, for optimal tissue function and resilience, the cells must be mechanically integrated to allow stresses to be spread across the whole tissue. Failure to do so can result in tissue fracture with consequences such as hemorrhage and septicemia ${ }^{14-17}$. Indeed, tissue fragility has been identified as a symptom in patients carrying mutations in intermediate filaments and desmosomal proteins ${ }^{18}$, adherens junction proteins and actin cytoskeletal regulators ${ }^{19-21}$, and as a result of bacterial pathogens targeting intercellular adhesions ${ }^{18}$. At timescales of seconds to minutes, the ability of living tissues to dissipate stresses decreases the risk of fracture $^{22}$, providing organisms with a protective mechanism against failure. Despite the importance of epithelial mechanics in barrier function, little is known about how epithelia dissipate stresses in response to extension.

In isolated cells, a rich phenomenology of rheological behaviours that operate at different timescales has been identified. At sub-second timescales, localised stress applied to the cell surface can be dissipated by redistribution of the fluid phase cytosol through the porous insoluble part of the cytoplasm ${ }^{23}$. At longer timescales, a scale-free power law rheology is observed ${ }^{23,24}$, which may stem from the large number of relaxation processes with different timescales operating in parallel in the cell ${ }^{25}$. Recent work has indicated the presence of a cut-off to the power law response imposed by the turnover rate of the actomyosin cytoskeleton ${ }^{26}$. 
In epithelia, the rheological behaviours observed in isolated cells may be influenced by the assembly of specialised intercellular junctions and junctional signaling ${ }^{27,28}$. Indeed, recent work has shown that adherens junctions, which link the actin cytoskeletons of adjacent cells, exhibit viscoelastic properties ${ }^{29}$. However, little is known about the stress response of cultured or embryonic epithelia to deformation - despite this being an important property of many normal tissues. Nor is it known which molecular mechanisms participate in the process. In part, this derives from the difficulties of measuring stress in epithelia that are mechanically coupled to a relatively thick and rigid extracellular matrix (ECM).

Here, to overcome this challenge, we study stress relaxation in cultured epithelial monolayers devoid of an ECM subjected to a physiologically relevant strain. Our analysis reveals that, at minute timescales, tissue rheology is dominated by the actomyosin cytoskeleton and that myosin contractility accelerates stress relaxation. By contrast, adherens junctions play little role in stress relaxation, acting as stable bridges connecting adjacent cells. As a consequence, the dynamics and amplitude of stress relaxation of an epithelial monolayer are similar to those of an isolated cell. Junctions linking cells within the tissue therefore enable them to act as an integrated mechanical unit.

\section{Results}

Monolayer stress relaxation is accompanied by a change in monolayer length

To investigate the response of epithelia to stress, we used monolayers of Madine-Darby Canine Kidney (MDCK II) cells devoid of a substrate and suspended between test rods ${ }^{14}$, 30. Under these conditions, all the stress in the system is borne by cells, simplifying interpretation and analysis (Fig S1). Suspended monolayers were subjected to a strain $\varepsilon_{0}=30 \%$ applied at a rate of $75 \% \cdot \mathrm{s}^{-1}$, within the linear regime of the stress-strain response for epithelial monolayers ${ }^{14}$ and consistent with deformations and rates observed in vivo under physiological conditions $7,10,11,31$. This $30 \%$ strain was then maintained for $\sim 130-140 \mathrm{~s}$ (Fig 1a,b, SI), while stress was monitored. Strikingly, under these conditions, $\sim 70 \%$ of the stress in monolayers was dissipated within $\sim 60 \mathrm{~s}$ (Fig 1c). Importantly, the behaviour of monolayers was reproducible over several cycles of stress relaxation. Moreover, cells maintained their characteristic apico-basal polarity and cytoskeletal organisation throughout ${ }^{14,32}$.

In the body, epithelia are generally bound to ECM. Therefore, to test the generality of the behaviour observed in suspended monolayers, we examined the response to deformation of a simple tissue comprising cells and ECM using Drosophila third larval instar wing imaginal discs. Wing discs consist of two epithelial layers, one columnar and one squamous, in apposition with one another and surrounded by a layer of ECM less than 1 $\mu \mathrm{m}$ thick ${ }^{33}$ (Fig S5a). Following application of a $20 \%$ strain to wing disc explants, stress rapidly dissipated (Fig 1d,e). However, the amplitude was smaller than in cultured monolayers ( $35 \%)$ and occurred over a longer duration (>100 s, Fig 1d,e, S5b). As ECM is known to turn over slowly ${ }^{34}$, these data indicate that larval epithelia bear at least a third of the total tissue stress and dynamically relax stress, consistent with previous qualitative observations ${ }^{35}$. 
In living tissues, stress relaxation can arise from a number of molecular and cellular processes. In our experiments, however, cellular processes, such as oriented cell division or neighbour exchange ${ }^{1,22,36}$, are unlikely to contribute to stress relaxation, since they occur over tens of minutes. In line with this, when we followed cells expressing ECadherin GFP at high magnification in cultured monolayers, images obtained immediately after extension and 30s later could be superimposed perfectly despite significant relaxation of stress (Fig 1C,f). Furthermore, cell areas and heights did not change during relaxation (Fig S2c,d). Nevertheless, while the application of stretch elongates the monolayer and no further changes are apparent while stretch is maintained, when the test rod is returned to its initial position at the end of the experiment $(\sim 130 \mathrm{~s}$ after application of stretch), the monolayer buckles, indicating an increase in length (Fig $\mathbf{1 g}$, Video $\mathbf{S 1}, \mathrm{n}=22 / 22$ monolayers). Thus, stress relaxation involves an increase in the length of the monolayer over time as the result of remodeling at the subcellular scale.

Monolayer stress relaxation is biphasic

We next characterised stress relaxation in detail. The process started immediately after extension and was biphasic, with a large amplitude fast relaxation occurring within the first $\sim 6 \mathrm{~s}$, followed by a smaller amplitude slow relaxation, which reached a plateau after $\sim 60 \mathrm{~s}$, as previously observed ${ }^{14}$ (Fig 1c, 2a). The presence of a plateau indicates that the material behaves like a solid at minute timescales. Examination of the relaxation curves in log-log and log-linear scales revealed that the dominant regime decays as a power law in the first phase and as an exponential in the second phase (Fig S3). We confirmed the power law nature of the first phase by performing stress relaxation experiments for a range of deformations (Fig S4c-f, SI). Based on these observations, the relaxation can be described by a function of the form $A t^{-\alpha} e^{-t / \tau}+B$ (Methods), where the kinetics of the first phase is characterised by the power law exponent $\alpha$ and the second phase by the time constant $\tau$. The parameter $B / \varepsilon_{0}$ is equivalent to an elasticity, and $A$ sets the amplitude of the relaxation. Using this empirical function to describe the data, monolayer stress relaxation curves could be fitted with high coefficients of determination $\left(r^{2}>0.8\right.$, $\mathrm{n}=17$ monolayers), without systematic bias in the residuals. The first phase had an exponent $\alpha=0.3 \pm 0.03$ and the second phase a time constant $\tau=14.9 \pm 5.8 \mathrm{~s}$. Relaxation in Drosophila wing discs displayed similar characteristics to cultured monolayers, although the parameters differed $(\alpha=0.16 \pm 0.05$ and $\tau=66.1 \pm 18.2 \mathrm{~s}$, $\mathrm{n}=12$ experiments, Fig S5c-h). Thus, larval and cultured epithelia display fluid-like properties at second timescales and solid-like properties at minute timescales.

To explore the robustness of this biphasic behaviour, we subjected cultured monolayers to a $30 \%$ strain applied at different strain rates and to different strains at a fixed $75 \% . \mathrm{s}^{-1}$ strain rate. In both cases, the experimental data was well described by our empirical fit function. For strains rates above $25 \% . \mathrm{s}^{-1}$, the fit parameters did not vary significantly, indicating that our initial experimental conditions $\left(30 \%\right.$ strain applied at $\left.75 \% . \mathrm{s}^{-1}\right)$ are close to a pure step strain for monolayers (Fig S6). However, for lower strain rates $\left(1 \% . s^{-1}\right.$ and $\left.5 \% . s^{-1}\right)$, the amplitude of relaxation was visibly reduced because relaxation and loading take place on similar time-scales (Fig S6a-b). To take loading into account, we fitted the temporal stress response $\sigma(t)$ for monolayers loaded at a $75 \% . \mathrm{s}^{-1}$ strain rate with a 
convolution of the loading rate $\dot{\varepsilon}(t)$ and a relaxation modulus $G(t): \sigma(t)=$ $\int_{0} G\left(t-t^{\prime}\right) \dot{\varepsilon}\left(t^{\prime}\right) d t^{\prime}$ with $G(t)=A^{\prime} t^{-\alpha} e^{-t / \tau}+B^{\prime}$ and $A=A^{\prime} . \varepsilon \quad{ }_{0}$ and $B=B^{\prime} . \varepsilon_{0}$ (Methods). The parameters obtained in this way did not differ significantly from those obtained considering a pure step in strain ( $p>0.1$ for all parameters, Table 2). We then used the parameters $\left(A^{\prime}, B^{\prime}, \alpha\right.$, and $\left.\tau\right)$ obtained for $75 \% . \mathrm{s}^{-1}$ strain rate to predict the relaxation of the tissue for other strain rates. Our predictions fell well within the range of experimental curves with no further fitting (Fig S7). In view of these results, we restricted ourselves to fitting stress relaxation with the function $\sigma(t)=A t^{-\alpha} e^{-t} / \tau+B$ in the rest of the study. In response to different stretch amplitudes $\varepsilon_{0}, \alpha$ did not vary, except for $50 \%$ strain when it decreased marginally, even though the effect was significant (Fig S8c). $A$ and $\tau$ increased significantly for $50 \%$ strain (Fig S8d,e). In addition, $A$ and $B$ displayed a trend of increasing with $\varepsilon_{0}$. Furthermore, when we plotted $\tau$ as a function of $\varepsilon_{0}$ for each monolayer separately, we found that $\tau$ increased with strain in all experiments with a slope significantly larger than zero (Fig $\mathbf{S 8 g}$, slope $=70.1 \pm 57.1 \mathrm{~s}, p<0.05$ ). The dependence of $\tau$ on $\varepsilon_{0}$ is surprising and may arise because the mechanisms dissipating stress occur at a rate that does not depend on strain.

The transition between the two relaxation phases occurs for $\mathrm{t} \sim 6 \mathrm{~s}$ (Fig S3c), a timescale short compared to that of biological processes involved in cell mechanics ${ }^{37}$. This suggests that passive, ATP-independent processes govern the power law behaviour of the first phase, while active ATP-dependent processes participate in the second phase. To test this hypothesis, we performed stress relaxation experiments on ATP-depleted monolayers. When we examined the relaxation curves for these tissues in log-log scales, they appeared linear (Fig 2b) and did not display the plateau at long time-scales characteristic of exponential relaxation (Fig 2a), as expected if the exponential relaxation phase was indeed ATP-dependent. The relaxation curves could be well fit with a single power law $A t^{-\alpha}(\alpha=0.16 \pm 0.03, \mathrm{n}=10$ monolayers, Fig 2b-c, S4a, Methods), showing that the second exponential phase disappeared or was severely delayed.

Monolayer stress relaxation depends on actomyosin not on junctional remodelling or intermediate filaments

As stress relaxation is accompanied by an increase in the length of the monolayer, we hypothesised that it may involve the dynamic turnover of the molecular constituents of cytoskeletal and adhesive structures. Based on previous work on the mechanics of single cells and tissues ${ }^{25,38}$, we decided to concentrate on the actin cytoskeleton, intermediate filaments, and the intercellular junctions that connect these structures (the adherens junctions and desmosomes).

To identify the key components of each of these structures in MDCK monolayers, we used mRNA sequencing (RNAseq) to quantify their relative abundance (SI). Although protein concentrations are not always directly correlated to mRNA transcript levels (because of differences in translation and protein degradation), they have nevertheless been shown to be good predictors of protein abundance ${ }^{39,40}$. Moreover, low mRNA transcript levels necessarily imply low protein abundance ${ }^{39}, 40$. Therefore, we classified 
proteins into categories reflecting the candidate subcellular structures (Fig S9a) and then selected proteins amongst the most abundant in each class for further examination.

We reasoned that only proteins that display significant turnover over the timescale of our experiments could significantly contribute to the relief of mechanical stress. To characterise turnover, we used Fluorescence Recovery After Photobleaching (FRAP) (SI). For this, we generated cell lines stably expressing GFP-tagged candidate proteins and confirmed their localisation to the relevant structures (Fig S10, SI). For each protein, we measured the percentage fluorescence recovery (mobile fraction) $100 \mathrm{~s}$ after photobleaching, because stress relaxation is complete within that timeframe. Proteins within the different candidate subcellular structures had strikingly distinct behaviours (Fig 2e,f, S9b,c, Table 1). Actin, myosin and crosslinkers were the most dynamic, with mobile fractions larger than 0.4 (Fig 2f, Table 1). In contrast, proteins of the cadherin-catenin complex, intermediate filaments and desmosomes had mobile fractions smaller than 0.1. Proteins involved in mechanotransduction exhibited intermediate levels of mobility (EPLIN and vinculin). Thus, the extent of recovery appears very different for different subcellular structures. Proteins of the actomyosin cytoskeleton (actin, myosin II and crosslinkers) have recoveries consistent with a potential role in stress relaxation. By contrast, proteins of the adherens junctions, intermediate filament networks, and desmosomes appear stable over the course of $100 \mathrm{~s}$.

To functionally test for a role for actomyosin in stress relaxation, we first depolymerised F-actin using latrunculin B (Fig 3a,b). Loss of F-actin led to a remarkable softening of the monolayer, as seen from the 10-fold lower stresses compared to DMSO control, and suggested that intermediate filaments bear little stress at this range of strain (Fig 3c,d). Furthermore, relaxation curves appeared linear in the logarithmic scale, pointing to a delay in the second phase or its complete abrogation (Fig 3d). Together, these data show that the actin cytoskeleton is responsible for the second phase of relaxation. As actinrelated proteins with fast turnover were found localised to both intercellular junctions and the submembranous cortex (Fig S10), this suggested that either of these actomyosin-rich structures may contribute to relaxation in the second phase.

Monolayer relaxation is significantly slowed by perturbing myosin contractility and actin polymerisation but not by depleting crosslinkers

Actin's function in cytoskeletal organisation is multi-faceted: it is the basic building block for generation of filamentous actin, F-actin serves as a scaffold for myosin contractility, and crosslinkers can modulate the network's mechanics.

Previous work has identified specific roles for actin networks generated through distinct nucleation pathways via the Arp2/3 complex and formins in epithelial tissues ${ }^{41-43}$. To determine the importance of actin organisation in monolayer stress relaxation, we inhibited the nucleation of actin filaments through the Arp2/3 complex using CK666, a drug that prevents Arp2/3 activation ${ }^{44}$, and through formins using SMIFH2, a drug that prevents barbed-end elongation via formins ${ }^{45}$. Formin inhibition led to a weakly significant increase in the relaxation time constant $\tau$ (Fig 3f). However, Arp2/3 complex inhibition did not significantly affect relaxation. 
Formin nucleated filaments also act as substrates for myosin contractility. Therefore, to investigate the role of active myosin, we treated the tissue with Y27632, a small molecule that inhibits Rho-kinase. Y27632 had a profound impact on monolayer stress relaxation. It significantly increased the relaxation time constant $\tau$, leading to curves that appeared more linear in logarithmic scale (Fig 3f, S12a), and reduced the elasticity $B / \varepsilon_{0}$ without affecting $A$ (Fig S12e,f), implying that myosin activity accelerates the return to mechanical equilibrium following extension.

Finally, we explored whether actin filament crosslinkers influence the dynamics of active relaxation by generating friction in the actomyosin network that slows relaxation, as seen in single cells ${ }^{26,46,47}$. To do so, we studied stress relaxation in monolayers expressing shRNAs targeting filamin A and $\alpha$-actinin 4 , the two most abundant actin crosslinkers identified in our RNAseq experiments (Fig S9a). Surprisingly, their depletion had no effect on the time constant $\tau$ or the elasticity $B / \varepsilon_{0}$ of monolayers (Fig 3e, S11). Thus, the dominant actin crosslinkers in the system do not play a role in setting the dynamics of monolayer stress relaxation.

Together, these results suggest that F-actin remodelling functions together with myosin II to ensure the rapid return of the monolayer to mechanical equilibrium following the application of strain.

Monolayer stress relaxation is similar to the relaxation of single rounded cells Interestingly, the amplitude and dynamics of stress relaxation in cultured monolayers appear similar to those previously reported for single cells ${ }^{26,48}$. To investigate these similarities further, we characterised the relaxation of isolated MDCK cells. In our experiments, we compressed single rounded cells with a tipless Atomic Force Microscope (AFM) cantilever to stretch the cell cortex in the free surfaces of the cell and monitored force relaxation $(\mathbf{F i g ~} 4 a)^{26}$. The transitory phase of force relaxation lasts $\sim 20 \mathrm{~s}$ and reports on dissipation mechanisms, while the plateau reports on cellular cortical tension ${ }^{26,49}$ (Fig 4b). Similar to monolayers, single cells relaxed following a power law at second timescales and an exponential at minute time-scales, consistent with previous work ${ }^{26}$ (Fig $\mathbf{4 b}$, S13a). Fitting these force relaxation curves with our empirical fit function yielded a time constant $\tau=13.4 \pm 15.0 \mathrm{~s}$, similar to monolayers $(p=0.18)$, and an exponent $\alpha=0.25 \pm 0.05$, weakly but significantly smaller than in monolayers $(p<0.05$, Fig 4c,d).

Given these results, we investigated if the second phase of stress relaxation in single cells was sensitive to the same perturbations as monolayers. Depletion of $\alpha$-actinin 4 did not affect the second phase of relaxation (Fig 4e, S13). Treatments with Y27632 and SMIFH2 both increased $\tau$, as in monolayers (Fig 4f), and decreased cellular cortical restoration force $C$ (Fig S14). Remarkably, these data reveal mechanistic similarities between stress relaxation in single rounded cells and in epithelial monolayers.

As the second phase of relaxation may stem from biochemical turnover of actomyosin, we characterised the turnover kinetics of actomyosin in single rounded cells using FRAP. 
Actin, myosins, and crosslinkers ( $\alpha$-actinin 4, filamin A) all localised to the cortex of rounded cells (Fig S15c). Furthermore, their mobile fractions and half times were similar to those measured in monolayers (Fig 4g, Fig S15a-b, Table 3). Together, these data indicate that single cells display similar stress relaxation kinetics to monolayers, are sensitive to similar perturbations, and have similar actomyosin turnover kinetics. This further suggests that stress relaxation may originate in the actin cortex, as it is the only actin- and myosin-rich cytoskeletal structure common to both single rounded cells and monolayers. In support of this hypothesis, the mobile fraction of actin in the apical cortex of cells within monolayers was not significantly different from that in single rounded cells ( $0.46 \pm 0.13, p=0.6$ compared to cortical actin in single cells, Fig $\mathbf{4 g}$ ).

A phenomenological model for monolayer stress relaxation Having shown that we could separate stress relaxation into a very rapid $(\mathrm{t}<6 \mathrm{~s})$ ATPindependent regime and an ATP- and actomyosin-dependent regime at longer timescales $(6<t<75 \mathrm{~s})$, we fitted the second phase of relaxation with rheological models to investigate the mechanical origins of the ATP-dependent regime and the dynamics of length change. Importantly, we modelled the monolayer as an integrated mechanical system, since our data showed that monolayer relaxation and single cell relaxation are highly similar. Based on our experimental data, we reasoned that ATP-dependent monolayer mechanics should consist of an elastic branch, that describes the response at minute-long timescales using a spring $\kappa$, placed in parallel with a viscous branch, that describes the transitory regime (Fig 5a, S16a).

Although a viscous branch consisting of a spring $\kappa_{M}$ in series with a dashpot with viscosity $\eta$ can fit the experimental stress evolution well $\left(r^{2}>0.8\right.$ for $88 \%$ of the relaxation curves) and can provide the evolution of the length of the monolayer (Fig S16, SI), the time constant of relaxation is fixed by material parameters $\tau_{M}=\eta / \kappa_{M}$ independently of strain, in contradiction with our observations (Fig S8g). As an alternative, we decided to use a model that considers length as an explicit variable ${ }^{50}$ because epithelia often change length during development ${ }^{51,52}$. Because of the role of myosin and changes in length during relaxation, we modelled the viscous behaviour using an active contractile element

which consists of a spring $\kappa_{A}$ subjected to a constant pre-strain $\varepsilon^{c}$ (Fig 5a). In response to an applied strain $\varepsilon_{0}$, this spring dynamically regulates its resting length $L(t)$ evolving as:

$\dot{L} / L=\gamma\left(\varepsilon^{e}(t)-\varepsilon^{c}\right) /\left|\varepsilon^{e}(\mathrm{t}=0 \mathrm{~s})-\varepsilon^{c}\right|$

with $\gamma$ a length-change rate. $\varepsilon^{e}(t)$ is the effective strain defined as:

$\varepsilon^{e}(t)=\left(l_{m}-L(t)\right) / L(t)$

with $l_{m}$ the actual length of the monolayer imposed by deformation ${ }^{51,53,54}$. Over time, the change in resting length $L(t)$ of the active branch dissipates stress with an exponential decay (equation 6 in Methods), similar to a Maxwell material (equation 14 in SI), converging towards the monolayer pre-stress $\sigma_{c}=\kappa_{A} \cdot \varepsilon^{c}$ (Methods). Therefore, after 
relaxation, stress in the monolayer reaches a plateau $\sigma_{\infty}=\kappa \varepsilon_{0}+\kappa_{A} \varepsilon^{c}$ with a characteristic time that increases with strain as $\tau_{\text {model }}=\varepsilon_{0} /\left[\gamma\left(1+\varepsilon_{0}\right)\right]$, as observed in experiments (Fig S8g, See analytical solution, Methods). In our model, the resting length of the active branch and the elastic branch of the system may be different because they originate from different cytoskeletal structures.

In experiments, we confirmed the presence of pre-stress in monolayers prior to extension with values $\sigma_{c} \sim 141 \mathrm{~Pa}(\mathrm{SI}$, Fig S17a,c), consistent with the presence of an active element. Following stress relaxation, the stress in the elastic branch $\left(\sigma_{\infty}-\sigma_{c}\right)$ appears to scale linearly with strain (Fig S17b), pointing to a solid, spring-like behaviour with $\kappa \sim 1006 \mathrm{~Pa}$ (Fig S17b). Next, we fitted the second phase of stress relaxation using the analytical solution in equation (7) to determine $\kappa_{A}$ and $\gamma$, using our measurements of $\sigma_{c}$ and $\kappa(\mathbf{F i g ~ S 1 7 c , g})$ together with the relationship $\sigma_{c}=\kappa_{A} \cdot \varepsilon^{c}$ (Fig 5b). Analytical curves fitted the experimental data well $\left(r^{2}>0.8\right.$ for $88 \%$ of the relaxation curves) without any systematic bias in the residuals, yielding $\kappa_{A} \sim 601 \mathrm{~Pa}, \varepsilon^{c}=0.26$ and $\gamma \sim 0.03 \mathrm{~s}^{-1}$ (Fig S17d-f). Thus, by introducing an active element that adjusts its resting length, we were able to reproduce monolayer stress relaxation in the second phase. Next, we tested the robustness of our fitting approach to variations in strain and strain rate. Neither variation in loading regime significantly changed the parameters $\kappa_{A}$ and $\gamma$, except at the slowest strain rate $\left(1 \% . \mathrm{S}^{-1}\right.$, Fig S18b,c, S19b,c). Deformations applied at $1 \% . \mathrm{S}^{-1}$ significantly decreased $\kappa_{A}$, because loading and exponential relaxation occur on similar time-scales. The characteristic time constants $\tau_{\text {model }}$ computed from the model correlated well with those determined from empirical fitting for all conditions (Fig 5f), further validating our approach. In our experiments, we noted that $\tau$ increased with applied strain (Fig S8d,g), a scaling that could be explicitly derived from our rheological model with no change in material parameters (Fig S19e, Methods). Furthermore, for small values of $\varepsilon_{0}$, the characteristic time of our model becomes linearly proportional to $\varepsilon_{0}: \tau_{\text {model }} \sim \varepsilon_{0} / \gamma$. Using this rough approximation, linear fits of our observations (Fig S8g) suggest that $\tau\left(\varepsilon_{0}\right)$ intercepts with the $y$-axis close to $0 \mathrm{~s}$ (intercept $=-5.5 \pm 11.4 \mathrm{~s}, p=0.31$ compared to zero), consistent with our model. The slopes of these linear fits predict $\gamma \sim 0.03 \pm 0.02 \mathrm{~s}^{-1}$, similar to the values obtained by fitting relaxation curves for 30\% strain (Fig S17e, $p=$ 0.28 ). The invariance of $\gamma$ with applied strain may signify its dependence on constitutive biochemical reactions (e.g. remodelling of actinous structures) and that these are not sensitive to strain.

Myosin contractility and formins contribute to relaxation by accelerating length change To understand the link between mechanical behaviour and biological mechanisms, we analysed perturbation experiments using our rheological model as a guide. Depolymerisation of F-actin by Latrunculin abrogated the second phase of relaxation and the solid-like behaviour at minute time-scales, suggesting that both branches of our model are actin-rich structures. Next, we measured changes to $\kappa$ and $\sigma_{c}$ from our experimental data and obtained values for $\kappa_{A}$ and $\gamma$ from curve fitting with the condition $\sigma_{c}=\kappa_{A} \cdot \varepsilon^{c}$. Both treatment with Y27632 and SMIFH2 decreased the length-change rate $\gamma$, but had no effect on the stiffness $\kappa_{A}$ of the active element (Fig $\mathbf{5 c , d}$ ). This suggested that both formins and myosin contractility contribute to stress relaxation by ensuring a rapid monolayer length change. Interestingly, Rho-kinase inhibition also decreased the pre-stress $\sigma_{c}$ and 
stiffness $\kappa$ of the elastic branch, while formin inhibition affected neither parameter (Fig 5e, S20a). Thus, myosin contributes to both the active and the elastic parts of the system, perhaps through its different functions (contractility and crosslinking) or because each branch represents a distinct actomyosin cytoskeletal structure.

Together, these results indicate that the pre-stress $\sigma_{c}$ depends on contractility alone, $\gamma$ depends on actin polymerisation and myosin, and $\kappa$ depends on myosin.

\section{Discussion}

Here, we characterise stress relaxation and the molecular turnover of the stress-bearing biological structures in single rounded cells and epithelial monolayers. Our data paint a picture in which intercellular junctions form stable interconnections between cells allowing the monolayer to behave as a single cell with its rheology controlled by cortical actomyosin. Together F-actin remodelling and myosin contractility endow the monolayer with solid-like mechanical properties at minute timescales, act as driving forces to reach a new mechanical steady-state following extension, and regulate the length of the monolayer.

\section{Monolayer stress relaxation dynamics}

When we examined the response of cultured epithelial monolayers to a step deformation, we found that most of the stress is dissipated at minute timescales and that relaxation can be described by a power law with an exponential cut-off at timescales larger than $\sim 10$ s. A similar response was observed in larval epithelia, however, the extent of stress relaxation was lower, likely because of the presence of ECM. Examination of the temporal evolution of cell morphology in monolayers revealed that dissipation occurred through dynamic molecular processes rather than cellular ones.

In cultured suspended monolayers, the first power law phase had an exponent $\alpha \sim 0.30$, consistent with previous reports for cell aggregates subjected to compression ${ }^{55}$, and was present even when ATP was depleted. Given the clear dependence of the second phase of relaxation on ATP, we focused on the contributions of subcellular structures known to play a role in cell and tissue mechanics such as the adherens junctions ${ }^{14,56,57}$, desmosomes ${ }^{58}$, intermediate filaments ${ }^{59,60}$ and actomyosin ${ }^{14,61-65}$. Stress relaxation within cytoskeletal and adhesive structures likely stems from molecular turnover of their constituents ${ }^{66}$. In support of this idea, intermediate filaments and desmosomes display very little turnover ${ }^{67-69}$ over the duration of stress relaxation (Fig 2, S9), similar to proteins of the cadherin-catenin adhesive complexes ${ }^{70}$, whereas proteins of the actomyosin cytoskeleton turn over extensively (Fig 2, S9). Furthermore, we found that treatments that target actomyosin lead to significant changes in stress relaxation (Fig 3, S12), suggesting that actomyosin governs monolayer rheology at second to minute timescales.

\section{Monolayer and single cells have similar rheology}

In line with actomyosin controlling rheology, stress relaxation in monolayers displayed many similarities to stress relaxation in single cells. This is surprising since the cytoskeletal organisation of single rounded cells and cells in epithelia differ markedly. 
Yet, when subjected to $30 \%$ deformation, both single cells and monolayers displayed an initial phase of relaxation following a power law before switching to an exponential decay reaching a plateau at minute-long timescales, consistent with previous reports ${ }^{26,48,71}$. The existence of a plateau indicates that both single cells and monolayers switch from a liquidlike behaviour at second timescales to a solid-like behaviour on minute-long timescales.

In the second phase of relaxation, stress in monolayers and single cells decayed with a time constant $\tau$ that was identical ( 14 s). In both situations, $\tau$ did not depend on crosslinkers but depended strongly on myosin contractility and formin activity. The similarities in the behaviour of monolayers and single cells implies that their rheology is governed by actomyosin structures present in both.

Molecular mechanisms controlling monolayer rheology

In both monolayers and single cells, the rheology depends strongly on actomyosin and we observed that cortical proteins turn over significantly over the time-scale of mechanical relaxation. In contrast, the adhesive structures present in monolayers remodel far less. One consequence of these clear differences in turnover extent is that adherens junctions form stable interconnections between cells allowing the monolayer to behave as a single cell with its rheology controlled by actomyosin. As the submembranous cortex is the only actomyosin-rich cytoskeletal structure common to both single rounded cells and cells within epithelial monolayers (Fig S10, S15) and as it turns over to a similar extent in both cellular configurations (Fig $\mathbf{4 g}$ ), this suggests that cortical actomyosin controls stress relaxation. This further implies that multicellular rheology in monolayers may be controlled by emergent properties of actomyosin gels at the molecular-scale ${ }^{72}, 73$, something that will form an interesting direction for future work.

Our mechanical model indicated that formins participated in setting the rate of length change $\gamma$, while inhibition of contractility affected $\gamma$, the pre-stress $\sigma_{c}$ and the stiffness of the elastic branch $\kappa$ (Fig 5, S20). The change in $\kappa$ is surprising and may indicate a role of myosins in crosslinking on minute time-scales. Indeed, although individual myosins within a mini-filament rapidly turn over (Fig 2f, $\mathbf{S 9 d}$ ), the mini-filament itself may persist far longer, providing stable crosslinking and contributing to network elasticity. In support of this hypothesis, measurements of the lifetime of myosin foci in the cortex of HeLa cells and blebs in melanoma cells indicate a characteristic life time of $\sim 130 \mathrm{~s}^{74,75}$, far longer than the turnover time of the myosins within the mini-filaments measured by FRAP (Fig S9d $)^{76}$.

Interestingly, the relaxation time constant $\tau$ increased with applied strain (Fig S8g) but why remodelling of the cortex should take longer for larger strain is unclear. Cortex remodelling requires a combination of nucleation of new actin filaments and depolymerisation ${ }^{77}$. When monolayers are stretched, their apical and basal areas increase ${ }^{14}$ potentially leading to a decrease in the concentration of actin nucleators at the membrane. As cortex thickness is regulated ${ }^{78,79}$, the lower nucleator concentration may lead to a longer remodeling time at high strain. Future experiments will be necessary to 
test this, although recent work has shown that cortical actin becomes limiting when cells are subjected to extreme strain ${ }^{80}$.

Monolayer length changes in response to application of stress

How stress is relaxed in isolated cells and monolayers remains poorly understood. Previous theoretical and experimental studies have suggested that changes in the resting length of cells and tissues may underlie stress relaxation ${ }^{51,53}$. In line with this, we showed that monolayer length increases in response to a sustained stretch (Fig 1 $\mathbf{g}$ ). This length change stems for a change in the length of the active branch of our model and appears to depend on formin-mediated polymerisation and myosin contractility, although the detailed molecular mechanism remains to be determined (Fig 5). Although length increase in the active branch of our model dissipates part of the stress that arises in response to monolayer extension, our model and experiments indicate that the elastic branch does not change length at minute time-scales. Further work will be necessary to determine the identity of the actomyosin structure that underlies this elastic-like behaviour. The realisation that some monolayer structures can change resting length over minute time-scales in response to stress may have important consequences for our understanding of developmental morphogenesis. Indeed, many developmental processes involve large tissue deformations in response to stress generated elsewhere in the embryo. Our results show that, in addition to cellular level processes (such as neighbour exchanges or oriented divisions) which occur over tens of minutes, molecular turnover can change cellular cortical area to dissipate stresses over minute long durations.

In summary, our data paint a picture in which actomyosin plays a central role in monolayer mechanics, rapidly acting to enable the tissue to reach a new mechanical steady-state, endowing the monolayer with solid-like mechanical properties at minute timescales, and regulating monolayer length. 


\section{Code availability}

Custom-written code used for data analysis are available from the authors upon request.

\section{Data availability}

All data supporting the conclusions are available from the authors on reasonable request.

\section{Acknowledgements}

The authors wish to acknowledge present and past members of the Charras, Baum, Kabla, and Muñoz labs for stimulating discussions. The authors acknowledge technical support from UCL Genomics for sequencing and analyzing total RNA data as well as from Julia Duque (LCN) for analysis of fluorescence intensity at junctions. N.K. was funded by the Rosetrees Trust, the UCL Graduate School, the EPSRC funded doctoral training program CoMPLEX, and the European Research Council (ERC-CoG MolCellTissMech, agreement 647186 to GC). N.K. was in receipt of a UCL Overseas Research Scholarship. N.K. was supported by the Prof Rob Seymour Travel Bursary Fund for research visits to Barcelona. J.F. and A.B. were funded by BBSRC grant (BB/M003280 and BB/M002578) to G.C. and A.K. J.J.M, N.A. and P.M. acknowledge the support of the Ministry of Economy, Industry and Competitiveness (MINECO) through Grants No. DPI201343727R, and DPI2016-74929-R and the Generalitat de Catalunya through Grant No. 2014-SGR-1471. N.A. was also financially supported by Universitat Politècnica de Catalunya (UPC) and Consorci Escola Industrial de Barcelona (CEIB) through Grant UPC-FPI 2012, and the European Research Council under the European Community's 7th Framework Programme (FP7/2007-2013)/ERC Grant Agreement No. 240487. P.M. was also supported by the European Molecular and Biology Organisation (EMBO) under grant ASTF 351-2016. RB is part of the EPSRC funded doctoral training program CoMPLEX. MD was funded by a Marie Skłodowska-Curie Horizon 2020 Individual Fellowship (MRTGS). A.Y. was supported by an HFSP Young Investigator award to G.C. (RGY 66/2013). A.H. was supported by BBSRC grant (BB/K013521) to G.C. and A.K. Y.F. was supported by Japan Society for the Promotion of Science (JSPS) Grant-in-Aid for Scientific Research on Innovative Areas 26114001, Grant-in-Aid for Scientific Research (A) 18H03994, Strategic Japanese-Swiss Science and Technology Program, AMED under Grant Number JP17ck0106361 and JP18cm0106234, SAN-ESU GIKEN CO. LTD, the Naito Foundation, and the Takeda Science Foundation. A.K. was supported by BBSRC grants (BB/K018175/1, BB/M003280 and BB/M002578). Y.M. is funded by a MRC Fellowship MR/L009056/1, a UCL Excellence Fellowship, and a NSFC International Young Scientist Fellowship 31650110472. B.B. is supported by UCL, a BBSRC project grant (BB/K009001/1) and a CRUK programme grant (17343). M.M. is supported by EPSRC (EP/K038656/1). G.C. is supported by a consolidator grant from the European Research Council (MolCellTissMech, agreement 647186). AFM equipment was purchased thanks to an ALERT16 grant from BBSRC to G.C.

\section{Author contributions}

N.K., A.H. and G.C. designed the experimental setup. N.K., A.K., B.B., M.M. and G.C. designed the experiments. N.K. carried out the relaxation experiments. G.C. carried out FRAP experiments and protein localisation experiments. A.Y. carried out western blot experiments. N.K. carried out most of the data and image analysis. J.F. carried out image analysis to measure pre-stress. A.B. and A.K. contributed to theoretical analysis. N.A., 
P.M. and J.J.M. designed the rheological model. J.J.M contributed to computational analysis. A.K., J.J.M and M.M. provided conceptual advice. R.B., M.D., Y.M. and N.K. carried out measurements on Drosophila wing disc explants. Y.F. provided cell lines. N.K., B.B. and G.C. wrote the manuscript. All authors discussed the results and manuscript.

\section{References}

1. Heisenberg, C.-P. \& Bellaïche, Y. Forces in Tissue Morphogenesis and Patterning. Cell 153, 948-962 (2013).

2. Martin, A.C., Gelbart, M., Fernandez-Gonzalez, R., Kaschube, M. \& Wieschaus, E.F. Integration of contractile forces during tissue invagination. The Journal of Cell Biology 188, 735-749 (2010).

3. Tschumperlin, D.J., Boudreault, F. \& Liu, F. Recent Advances and New Opportunities in Lung Mechanobiology. Journal of biomechanics 43, 99 (2010).

4. Califano, J.P. \& Reinhart-King, C.A. Exogenous and endogenous force regulation of endothelial cell behavior. Journal of Biomechanics 43, 79-86 (2010).

5. Blanchard, G.B. et al. Tissue tectonics: morphogenetic strain rates, cell shape change and intercalation. Nature Methods 6, 458-464 (2009).

6. He, Z., Ritchie, J., Grashow, J.S., Sacks, M.S. \& Yoganathan, A.P. In Vitro Dynamic Strain Behavior of the Mitral Valve Posterior Leaflet. Journal of Biomechanical Engineering 127, 504-511 (2005).

7. Sacks, M.S. et al. In-Vivo Dynamic Deformation of the Mitral Valve Anterior Leaflet. The Annals of Thoracic Surgery 82, 1369-1377 (2006).

8. Perlman, C.E. \& Bhattacharya, J. Alveolar expansion imaged by optical sectioning microscopy. Journal of Applied Physiology (Bethesda, Md. : 1985) 103, 1037-1044 (2007).

9. Padala, M. et al. Mechanics of the mitral valve strut chordae insertion region. Journal of Biomechanical Engineering 132, 081004 (2010).

10. Rausch, M.K. et al. In Vivo Dynamic Strains of the Ovine Anterior Mitral Valve Leaflet. Journal of biomechanics 44, 1149-1157 (2011).

11. Maiti, R. et al. In vivo measurement of skin surface strain and sub-surface layer deformation induced by natural tissue stretching. Journal of the Mechanical Behavior of Biomedical Materials 62, 556-569 (2016).

12. Korkmaz, I. \& Rogg, B. A simple fluid-mechanical model for the prediction of the stress-strain relation of the male urinary bladder. Journal of Biomechanics 40, 663668 (2007).

13. Obropta, E.W. \& Newman, D.J. in 2016 IEEE Aerospace Conference 1-9 (2016).

14. Harris, A.R. et al. Characterizing the mechanics of cultured cell monolayers. Proceedings of the National Academy of Sciences of the United States of America 109, 16449-16454 (2012).

15. Suki, B. \& Hubmayr, R. Epithelial and endothelial damage induced by mechanical ventilation modes. Current Opinion in Critical Care 20,17-24 (2014).

16. Casares, L. et al. Hydraulic fracture during epithelial stretching. Nature Materials 14, 343-351 (2015).

17. Jufri, N.F., Mohamedali, A., Avolio, A. \& Baker, M.S. Mechanical stretch: physiological and pathological implications for human vascular endothelial cells. Vascular Cell 7, 8 (2015). 
18. Getsios, S., Huen, A.C. \& Green, K.J. Working out the strength and flexibility of desmosomes. Nature Reviews. Molecular Cell Biology 5, 271-281 (2004).

19. Levine, E., Lee, C.H., Kintner, C. \& Gumbiner, B.M. Selective disruption of Ecadherin function in early Xenopus embryos by a dominant negative mutant. Development 120, 901-909 (1994).

20. Maître, J.-L. et al. Adhesion Functions in Cell Sorting by Mechanically Coupling the Cortices of Adhering Cells. Science 338, 253-256 (2012).

21. Tang, V.W. \& Brieher, W.M. FSGS3/CD2AP is a barbed-end capping protein that stabilizes actin and strengthens adherens junctions. The Journal of Cell Biology 203, 815-833 (2013).

22. Wyatt, T., Baum, B. \& Charras, G. A question of time: tissue adaptation to mechanical forces. Current Opinion in Cell Biology 38, 68-73 (2016).

23. Moeendarbary, E. et al. The cytoplasm of living cells behaves as a poroelastic material. Nature Materials 12, 253-261 (2013).

24. Trepat, X. et al. Universal physical responses to stretch in the living cell. Nature 447, 592-595 (2007).

25. Kollmannsberger, P. \& Fabry, B. Linear and Nonlinear Rheology of Living Cells. Annual Review of Materials Research 41, 75-97 (2011).

26. Fischer-Friedrich, E. et al. Rheology of the Active Cell Cortex in Mitosis. Biophysical Journal 111, 589-600 (2016).

27. Priya, R. et al. Feedback regulation through myosin II confers robustness on RhoA signalling at E-cadherin junctions. Nat Cell Biol 17, 1282-1293 (2015).

28. Charras, G. \& Yap, A.S. Tensile Forces and Mechanotransduction at Cell\&\#x2013;Cell Junctions. Current Biology 28, R445-R457 (2018).

29. Bambardekar, K., Clément, R., Blanc, O., Chardès, C. \& Lenne, P.-F. Direct laser manipulation reveals the mechanics of cell contacts in vivo. Proceedings of the National Academy of Sciences 112, 1416-1421 (2015).

30. Harris, A.R. et al. Generating suspended cell monolayers for mechanobiological studies. Nature protocols 8, 2516-2530 (2013).

31. Roan, E. \& Waters, C.M. What do we know about mechanical strain in lung alveoli? American Journal of Physiology - Lung Cellular and Molecular Physiology 301, L625-L635 (2011).

32. Wyatt, T.P.J. et al. Emergence of homeostatic epithelial packing and stress dissipation through divisions oriented along the long cell axis. Proceedings of the National Academy of Sciences of the United States of America 112, 5726-5731 (2015).

33. Pastor-Pareja, J.C. \& Xu, T. Shaping Cells and Organs in Drosophila by Opposing Roles of Fat Body-Secreted Collagen IV and Perlecan. Developmental cell 21, 245-256 (2011).

34. Humphrey, J.D., Dufresne, E.R. \& Schwartz, M.A. Mechanotransduction and extracellular matrix homeostasis. Nature reviews. Molecular cell biology 15, 802812 (2014).

35. Duda, M. et al. Polarization of Myosin II refines tissue material properties to buffer mechanical stress. Dev Cell (2019), in press.

36. Lecuit, T. \& Yap, A.S. E-cadherin junctions as active mechanical integrators in tissue dynamics. Nat Cell Biol 17, 533-539 (2015). 
37. Gallet, F., Arcizet, D., Bohec, P. \& Richert, A. Power spectrum of out-of-equilibrium forces in living cells: amplitude and frequency dependence. Soft Matter 5, 29472953 (2009).

38. Khalilgharibi, N., Fouchard, J., Recho, P., Charras, G. \& Kabla, A. The dynamic mechanical properties of cellularised aggregates. Current Opinion in Cell Biology 42, 113-120 (2016).

39. Ramakrishnan, S.R. et al. Integrating shotgun proteomics and mRNA expression data to improve protein identification. Bioinformatics 25, 1397-1403 (2009).

40. Vogel, C. \& Marcotte, E.M. Insights into the regulation of protein abundance from proteomic and transcriptomic analyses. Nat Rev Genet 13, 227-232 (2012).

41. Cavey, M., Rauzi, M., Lenne, P.-F. \& Lecuit, T. A two-tiered mechanism for stabilization and immobilization of E-cadherin. Nature 453, 751-756 (2008).

42. Verma, S. et al. A WAVE2-Arp2/3 actin nucleator apparatus supports junctional tension at the epithelial zonula adherens. Molecular biology of the cell 23, 46014610 (2012).

43. Kovacs, E.M. et al. N-WASP regulates the epithelial junctional actin cytoskeleton through a non-canonical post-nucleation pathway. Nature Cell Biology 13, 934 (2011).

44. Nolen, B.J. et al. Characterization of two classes of small molecule inhibitors of Arp2/3 complex. Nature 460, 1031-1034 (2009).

45. Rizvi, S.A. et al. Identification and Characterization of a Small Molecule Inhibitor of Formin-Mediated Actin Assembly. Chemistry \& Biology 16, 1158-1168 (2009).

46. Natsuhiko, Y. \& Philippe, M. Contraction of cross-linked actomyosin bundles. Physical Biology 9, 046004 (2012).

47. Schiffhauer, E.S. et al. Mechanoaccumulative Elements of the Mammalian Actin Cytoskeleton. Current Biology 26, 1473-1479 (2016).

48. Desprat, N., Guiroy, A. \& Asnacios, A. Microplates-based rheometer for a single living cell. Review of Scientific Instruments 77, 055111 (2006).

49. Fischer-Friedrich, E., Hyman, A.A., Jülicher, F., Müller, D.J. \& Helenius, J. Quantification of surface tension and internal pressure generated by single mitotic cells. Scientific Reports 4, 6213 (2014).

50. Munoz, J.J. \& Albo, S. Physiology-based model of cell viscoelasticity. Phys Rev E Stat Nonlin Soft Matter Phys 88, 012708 (2013).

51. Doubrovinski, K., Swan, M., Polyakov, O. \& Wieschaus, E.F. Measurement of cortical elasticity in Drosophila melanogaster embryos using ferrofluids. Proceedings of the National Academy of Sciences of the United States of America 114, 1051-1056 (2017).

52. Clement, R., Dehapiot, B., Collinet, C., Lecuit, T. \& Lenne, P.F. Viscoelastic Dissipation Stabilizes Cell Shape Changes during Tissue Morphogenesis. Curr Biol 27, 3132-3142.e3134 (2017).

53. Muñoz, J.J. \& Albo, S. Physiology-based model of cell viscoelasticity. Physical Review E 88, 012708 (2013).

54. Clément, R., Collinet, C., Dehapiot, B., Lecuit, T. \& Lenne, P.-F. Viscoelastic dissipation stabilizes cell shape changes during tissue morphogenesis. bioRxiv (2017). 
55. Forgacs, G., Foty, R.A., Shafrir, Y. \& Steinberg, M.S. Viscoelastic properties of living embryonic tissues: a quantitative study. Biophysical Journal 74, 2227-2234 (1998).

56. Harris, A., Daeden, A. \& Charras, G. Formation of adherens junctions leads to the emergence of a tissue-level tension in epithelial monolayers. Journal of Cell Science 127, 2507-2517 (2014).

57. Gonzalez-Rodriguez, D. et al. Detachment and fracture of cellular aggregates. Soft Matter 9, 2282-2290 (2013).

58. Johnson, J.L., Najor, N.A. \& Green, K.J. Desmosomes: Regulators of Cellular Signaling and Adhesion in Epidermal Health and Disease. Cold Spring Harbor Perspectives in Medicine 4 (2014).

59. Wang, N. \& Stamenović, D. Contribution of intermediate filaments to cell stiffness, stiffening, and growth. American Journal of Physiology - Cell Physiology 279, C188 (2000).

60. Ramms, L. et al. Keratins as the main component for the mechanical integrity of keratinocytes. Proceedings of the National Academy of Sciences 110, 1851318518 (2013).

61. Sato, M., Levesque, M.J. \& Nerem, R.M. Micropipette aspiration of cultured bovine aortic endothelial cells exposed to shear stress. Arteriosclerosis, Thrombosis, and Vascular Biology 7, 276-286 (1987).

62. Fletcher, D.A. \& Mullins, R.D. Cell mechanics and the cytoskeleton. Nature 463, 485-492 (2010).

63. Bonnet, l. et al. Mechanical state, material properties and continuous description of an epithelial tissue. Journal of The Royal Society Interface 9, 2614-2623 (2012).

64. Martin, A.C., Kaschube, M. \& Wieschaus, E.F. Pulsed actin-myosin network contractions drive apical constriction. Nature 457, 495 (2009).

65. Machado, P.F. et al. Emergent material properties of developing epithelial tissues. BMC Biology 13, 98 (2015).

66. Kim, T., Gardel, M.L. \& Munro, E. Determinants of fluidlike behavior and effective viscosity in cross-linked actin networks. Biophysical journal 106, 526-534 (2014).

67. Windoffer, R., Beil, M., Magin, T.M. \& Leube, R.E. Cytoskeleton in motion: the dynamics of keratin intermediate filaments in epithelia. The Journal of Cell Biology 194, 669-678 (2011).

68. Moch, M., Herberich, G., Aach, T., Leube, R.E. \& Windoffer, R. Measuring the regulation of keratin filament network dynamics. Proceedings of the National Academy of Sciences 110, 10664-10669 (2013).

69. Gloushankova, N.A., Wakatsuki, T., Troyanovsky, R.B., Elson, E. \& Troyanovsky, S.M. Continual assembly of desmosomes within stable intercellular contacts of epithelial A-431 cells. Cell and Tissue Research 314, 399-410 (2003).

70. Yamada, S., Pokutta, S., Drees, F., Weis, W.I. \& Nelson, W.J. Deconstructing the cadherin-catenin-actin complex. Cell 123, 889-901 (2005).

71. Desprat, N., Richert, A., Simeon, J. \& Asnacios, A. Creep function of a single living cell. Biophysical Journal 88, 2224-2233 (2005).

72. Kruse, K., Joanny, J.F., Jülicher, F., Prost, J. \& Sekimoto, K. Generic theory of active polar gels: a paradigm for cytoskeletal dynamics. The European Physical Journal E 16, 5-16 (2005). 
73. Prost, J., Julicher, F. \& Joanny, J.F. Active gel physics. Nat Phys 11, 111-117 (2015).

74. Nie, W., Wei, M.-T., Ou-Yang, D.H., Jedlicka, S.S. \& Vavylonis, D. Formation of contractile networks and fibers in the medial cell cortex through myosin-II turnover, contraction, and stress-stabilization. Cytoskeleton (Hoboken, N.J.) 72, 29-46 (2015).

75. Charras, G.T., Coughlin, M., Mitchison, T.J. \& Mahadevan, L. Life and Times of a Cellular Bleb. Biophysical Journal 94, 1836-1853 (2008).

76. Fritzsche, M., Lewalle, A., Duke, T., Kruse, K. \& Charras, G. Analysis of turnover dynamics of the submembranous actin cortex. Molecular Biology of the Cell 24, 757-767 (2013).

77. Salbreux, G., Charras, G. \& Paluch, E. Actin cortex mechanics and cellular morphogenesis. Trends in Cell Biology 22, 536-545 (2012).

78. Biro, M. et al. Cell cortex composition and homeostasis resolved by integrating proteomics and quantitative imaging. Cytoskeleton (Hoboken) 70, 741-754 (2013).

79. Chugh, P. et al. Actin cortex architecture regulates cell surface tension. Nat Cell Biol 19, 689-697 (2017).

80. Latorre, M. \& Humphrey, J.D. A Mechanobiologically Equilibrated Constrained Mixture Model for Growth and Remodeling of Soft Tissues. Zeitschrift fur angewandte Mathematik und Mechanik 98, 2048-2071 (2018). 


\section{Methods}

Cell culture and generation of cell lines

MDCK II cells were cultured at $37 \mathrm{C}$ in an atmosphere of $5 \% \mathrm{CO}_{2}$ in air in high glucose DMEM (ThermoFisher) supplemented with 10\% FBS (Sigma) and 1\% penicillinstreptomycin (ThermoFisher). Mechanical experiments and imaging were performed in Leibovitz's L15 without phenol red (ThermoFisher) supplemented with 10\% FBS.

In order to visualise the junctional and cytoskeletal structures, as well as to determine the turnover kinetics of various proteins, stable lines of MDCK II cells expressing the following proteins were used: E-Cadherin GFP, actin GFP, Lifeact-GFP, $\alpha$-catenin GFP, $\beta$-catenin GFP, vinculin GFP, EPLIN GFP, $\alpha$-actinin 1 GFP, $\alpha$-actinin 4 GFP, filamin A GFP, vimentin GFP, keratin 18 GFP, desmoplakin GFP, NMHCIIA GFP and NMHCIIB GFP. Cell lines expressing E-Cadherin GFP, Lifeact-GFP and keratin 18 GFP were described in Harris et al. ${ }^{14}$. Other cell lines were generated by linearisation of plasmids encoding the FP tagged protein of interest with the appropriate restriction enzyme. The following plasmids were used: $\alpha$-catenin GFP (a kind gift of Dr E Sahai, the Francis Crick Institute, UK), $\beta$-catenin GFP (a kind gift of Dr Beric Henderson, University of Sydney, Australia), vinculin GFP (a kind gift of Prof Susan Craig, Johns Hopkins University, USA), EPLIN GFP (a kind gift of Prof Elizabeth Luna, University of Massachusetts, USA, Addgene plasmid 40947), $\alpha$-actinin 1 GFP $81, \alpha$-actinin 4 GFP (a kind gift of Prof Doug Robinson, Johns Hopkins University, USA), filamin A GFP (a kind gift of Dr Paul Shore, University of Manchester, UK), vimentin GFP (a kind gift of Prof Robert Goldman, Northwestern University, USA), desmoplakin GFP (a kind gift of Prof Kathleen Green, Northwestern University, USA, Addgene plasmid 32227), NMHCIIA GFP and NMHCIIB GFP (both kind gifts of Dr Robert Adelstein, National Heart, Lung and Blood Institute, USA, Addgene plasmids 11347 and 11348). The cell line expressing actin GFP was generated by inserting actin-GFP into a retroviral vector (pLPCX, Takara Clontech), generating retrovirus as described in Harris et al. ${ }^{14}$, and transducing it into MDCK cells. To create all other stable cell lines, the plasmid of interest was first linearised with the appropriate restriction enzyme and then transfected into wild type MDCK II cells using electroporation (Lonza CLB). $\sim 10^{6}$ cells were transfected with $10 \mu \mathrm{g}$ (NMHCIIA-GFP, NMHCIIB-GFP) or $2 \mu \mathrm{g}$ (all other plasmids) of cDNA according to manufacturer's instructions and then selected with antibiotics for 2 weeks. In order to achieve a homogenous level of fluorescence expression, cells were sorted using flow cytometry. Cells expressing E-Cadherin GFP were cultured in presence of $250 \mathrm{ng}^{-\mathrm{ml}^{-1}}$ puromycin. Cells expressing actin GFP were selected in presence of $1 \mu \mathrm{g} \cdot \mathrm{ml}^{-1}$ puromycin. All other cell lines were selected in presence of 1 mg. $\mathrm{ml}^{-1} \mathrm{G} 418$.

To study the role of crosslinkers, cell lines stably expressing shRNA targeting filamin A and $\alpha$-actinin 4 were used. Filamin A shRNA was expressed in a tetracycline-inducible manner 82 . These cells were cultured in presence of $5 \mu \mathrm{g} \cdot \mathrm{ml}^{-1}$ blasticidin and $800 \mu \mathrm{g} \cdot \mathrm{ml}^{-1}$ G418. To induce expression of shRNA, cells were incubated in presence of $2 \mu \mathrm{g} \cdot \mathrm{ml}^{-1}$ doxycycline for $72 \mathrm{~h}$ prior to the experiments. Plasmids encoding non-silencing shRNA and shRNA targeting $\alpha$-actinin 4 were a kind gift from Prof Bill Brieher (University of Illinois Urbana-Champaign, USA). Following linearisation of the plasmids, stable cell lines expressing control shRNA and $\alpha$-actinin 4 shRNA were generated by transfecting the 
plasmids into wild type cells using electroporation (Lonza CLB) as described above. Control and $\alpha$-actinin shRNA lines were amplified and selected in presence of $4 \mu \mathrm{g} \cdot \mathrm{ml}^{-1}$ puromycin. Protein depletion was ascertained using Western blotting.

Generating suspended cell monolayers

Suspended cell monolayers were generated as described by Harris et al. ${ }^{14}$, 30. Further information is provided in SI.

Mechanical testing procedure

The mechanical testing setup was assembled on top of an inverted microscope (Olympus IX-71) (Fig S1a). First, the petri dish containing the stress measurement device was secured on the microscope stage with 4 pieces of plasticine. The force transducer (SIKG7A, World Precision Instruments) with a tweezer-shaped mounting hook (SI-TM5KG7A-97902, World Precision Instruments) was mounted on a 3D motorised micromanipulator (Physik Instrumente) with a custom-made adaptor. The fixed rod of the device was held with the arm of a 3D manual micromanipulator (Fig S1a), while the top Tygon section of the flexible rod was held with the tip of the force transducer (Fig S1c). Both motorised and manual micromanipulators were equipped with a magnetic plate that secured them to the custom-made metal stage of the microscope.

Using the motorised micromanipulator, the monolayers could be extended to different strains with controlled strain rates. Extended monolayers exerted restoring forces on the flexible rod, causing the transducer tip to bend. The extent of bending was translated into a voltage value that was converted into a digital signal using a data acquisition system (USB-1608G, Measurement Computing) and recorded onto a computer. Both the data acquisition system and the motorised micromanipulator were controlled with a customwritten code in Labview. The monolayer and the transducer tip were imaged every $0.5 \mathrm{~s}$ using a $2 \times$ objective $(2 \times$ PLN, Olympus).

The mechanical testing procedure consisted of several steps:

- Initial approach: The tip of the force transducer was initially brought into contact with the Tygon tubing and then positioned such that the left tweezer arm was out of contact but within $50 \mu \mathrm{m}$ distance from the Tygon tubing. This enabled identification of the contact point of the transducer tip with the device during the mechanical testing procedure (Fig S1c).

- Preconditioning: The monolayers were subjected to 8 cycles of loading to a $30 \%$ target strain at a $1 \% \cdot \mathrm{s}^{-1}$ strain rate. This ensured breakage of any residual collagen attached to the monolayer (especially close to the rods), as well as causing the samples to evolve into a "preconditioned" state, where the slope of the stress-strain curve did not change in successive cycles. Hence, several experiments could be conducted on the same sample with a high degree of reproducibility.

- Stress relaxation experiments: The monolayers were extended to $30 \%$ strain at a $75 \% . \mathrm{s}^{-1}$ strain rate and then kept at a fixed $30 \%$ strain for $\sim 130-140 \mathrm{~s}$. The micromanipulator was then returned to the position it occupied before stretch (Fig 1a). This released the monolayers and they were left unstretched for $\sim 130-140 \mathrm{~s}$ to recover before performing another stress relaxation experiment. This stress 
relaxation experiment was repeated 3 times on each monolayer.

- Loading until failure: The monolayers were extended until failure at $1 \% . s^{-1}$ strain rate. After rupturing the monolayer, the flexible rod was returned to its initial position.

- Calibration of the device: To allow conversion from voltages to force, the device was calibrated. For this, the wire was extended at the same rate and to the same extent as in the cycling experiments. This was repeated 5 times. The length of the wire $L_{w}$ was measured using a Canon FD macro-lens (Canon, Surrey, UK) interfaced to a Hamamatsu EMCCD camera (Hamamatsu Orca ER, Hamamatsu UK, Hertfordshire, UK) (Fig S1b). Together with the mechanical properties of the wire, knowledge of $L_{w}$ enable determination of the bending stiffness of the wire and hence the force applied for a given deflection.

A detailed description of the procedure for conversion of voltages to forces is given in Supplementary Information.

Drosophila wing disc mechanical testing

The stress measurement devices were similar to those used for monolayer mechanical testing (see SI) with the following modifications: a stiffer NiTi wire $(0.3 \mathrm{~mm}$ diameter) was used and glass coverslips were glued onto the test rods for attachment of the wing discs. Prior to experiments, the glass coverslips were coated with CellTak (Corning) and left to dry. The dish containing the device was then filled with ex vivo culture medium ${ }^{83,84}$. Next, between 4-6 wing discs were dissected from $3^{\text {rd }}$ instar ( 120 h AEL) wild-type ( $\left.y w ; ;\right)$ larvae, transferred to the dish and placed onto the CellTak, bridging the gap between the two coverslips. The discs were positioned such that their anterior and posterior extremities were attached to the CellTak.

Mechanical testing was carried out as for monolayers with a few modifications. Initially, the wing disc was preconditioned by stretching by $0.15 \mathrm{~mm}$ at a rate of $0.01 \mathrm{~mm} . \mathrm{s}^{-1}$. This was repeated 5 times. Then the disc was rested for $2.5 \mathrm{~min}$ to allow full relaxation. The stretching experiments were conducted by stretching the discs by $0.15 \mathrm{~mm}$ at a rate of 1 $\mathrm{mm} . \mathrm{s}^{-1}$. The wire was calibrated by stretching by $0.8 \mathrm{~mm}$ at $0.01 \mathrm{~mm} . \mathrm{s}^{-1}$ rate. This was repeated 5 times.

Single rounded cell mechanical testing procedure

Prior to experiments, MDCK cells were trypsinised and plated sparsely in a glass bottomed Petri dish (35 mm diameter, WPI) and left to settle for 10-30 minutes. The experiments were conducted while the cells remained rounded and before they started to spread.

Force relaxation measurements were conducted using a CellHesion 200 Atomic Force Microscope (JPK Instruments, Berlin, Germany) mounted on a scanning laser confocal microscope (Olympus IX81 with a FV1000 confocal head) and tipless silicon SPM-Sensor cantilevers (ARROW-TL1Au-50, Nano World) with nominal spring constant of $0.03 \mathrm{~N} \cdot \mathrm{m}^{-1}$. The sensitivity of each cantilever was measured from the slope of a force-displacement curve acquired on a glass coverslip, and the spring constant was calibrated using the thermal noise fluctuation method. The spring constants estimated for each experiment 
ranged between $0.055-0.06 \mathrm{~N} \cdot \mathrm{m}^{-1}$.

Before conducting force relaxation experiments, force-displacement curves were acquired on the cell and a glass region close to it. Using these two curves, we estimated the cell height as the difference between the cantilever contact with the cell and glass. Next, we estimated the target force required to indent the cell by $\sim 30 \%$. Finally, force relaxation curves were acquired by indenting the cell to the target force of 5-40 $\mathrm{nN}$ at a rate of $75 \% . \mathrm{s}^{-1}$ and maintaining the cantilever at a constant height for $150 \mathrm{~s}$ while the force was recorded.

Analysis of the relaxation curves

To analyse the response of monolayers to a step deformation, the first $75 \mathrm{~s}$ of the stress relaxation curves were fitted with a function consisting of a power law with an exponential cut-off:

$$
\sigma(t)=A t^{-\alpha} e^{-\frac{t}{\tau}}+B
$$

The fitting procedure was as follows. First, the initial conditions for the fitting were determined. $B$ was the residual stress after the curves plateaued and was defined as the average of stress between $70 \mathrm{~s}<\mathrm{t}<75 \mathrm{~s}$. $A+B$ was defined as the initial stress at the second timepoint ( $t=0.150 \mathrm{~s}$ ) after the step deformation (Fig S3a). The first timepoint after application of the step deformation was ignored to allow the calculations to be performed on a logarithmic scale. To estimate $\alpha$, the first $5 \mathrm{~s}$ of the curves were used. In practice, $\sigma(t<5 s)-B$ was plotted as a function of time on a logarithmic scale and fitted with a line, with $\alpha$ being the slope of this line (Fig S3b). To estimate $\tau, \sigma(5<\mathrm{t}<20 \mathrm{~s})-$ $B$ was plotted in a semi-logarithmic scale and fitted with a line, with $\tau$ being the slope of this line (Fig S3c). Each experimental relaxation curve was fitted using equation (1), with the free parameters $A, \alpha$ and $\tau$. $B$ was also allowed to vary by $15 \%$ to optimise the fits (Fig S3d). The trust-region-reflective least squares algorithm, a built-in Matlab fitting procedure, was used for the fitting. The fitting was performed for the three individual repeats of the stress relaxation experiments on each monolayer. The fitted values obtained from the three repeats were then averaged to obtain a single value for each parameter.

For ATP depletion experiments, we followed the same general procedure except that we fitted experimental curves with a function of the form $A t^{-\alpha}$.

The same procedures were also followed to fit the relaxation curves of single rounded cells and larval wing discs. For single cell relaxation curves, since the residual force reports on cortical restoration force, we have denoted it a different parameter $C$, which was estimated and fitted similar to $B$ for monolayers. Due to the slower relaxation of the larval wing discs, we fitted the first $120 \mathrm{~s}$ of the relaxation curves and $B$ was defined as the average of stress between $115<\mathrm{t}<120 \mathrm{~s}$.

The goodness of fit was determined using the coefficient of determination $r^{2}$ and curves with $r^{2}<0.80$ were excluded from further analysis. This represented less than $3 \%$ of experimental curves acquired. Outliers were determined as described in the statistical 
analysis section and the curves for which either of the two parameters $\alpha$ and $\tau$ were outliers were not included for statistical analysis. On average, less than $13 \%$ of the data was excluded from analysis.

Analysis of the relaxation curves taking loading into account The relaxation modulus $\mathrm{G}(\mathrm{t})$ describes the behaviour of a viscoelastic material and is obtained from the response of the material to a step in strain. This ideal loading condition cannot be achieved experimentally. In practice, strain is applied with a constant strain

rate $\dot{\varepsilon}$ until reaching the target strain $\varepsilon_{0}$, after which strain is kept constant. Thus, the temporal evolution of stress $\sigma(t)$ in the material is given by the convolution between the relaxation modulus and the derivative of the strain:

$$
\sigma(t)=\int_{0}^{t} G\left(t-t^{\prime}\right) \frac{d \varepsilon\left(t^{\prime}\right)}{d t} d t^{\prime}
$$

where the relaxation modulus is of the form $G(t)=A^{\prime} t^{-\alpha} e^{-\frac{t}{\tau}}+B^{\prime}$ and $A^{\prime}$ and $B^{\prime}$ are related to $A$ and $B$ in equation (1) as follows: $A=\varepsilon_{0} A^{\prime}$ and $B=\varepsilon_{0} B^{\prime}$.

We fitted the relaxation responses of monolayers loaded at a $75 \% . \mathrm{s}^{-1}$ strain rate, using equation (2). The response of the material predicted using the average parameters extracted from fitting with equation (2) (Fig S7a, black line) are in good agreement with those obtained approximating our experimental conditions to an ideal step strain (Fig S7a, red line). Statistical comparisons indicate that the parameters obtained through both methods are not significantly different (Table S2). Thus, the relaxation responses obtained for strain rates of $75 \% . \mathrm{s}^{-1}$ can be approximated by an ideal step strain.

Confocal microscopy for cell area and height measurements

High magnification imaging devices were prepared as described in SI. Images for cell area measurements were obtained using either a scanning laser confocal microscope (Olympus IX-81 with an FV-1000 confocal head) with a 20x objective (UPLSAPO, Olympus, N.A. $=0.75$, working distance: $0.6 \mathrm{~mm}$ ) or a spinning disc confocal microscope (CS22, Yokogawa) with a 40× objective (UPLSAPO, Olympus, N.A. $=0.9$, working distance: $0.18 \mathrm{~mm}$ ) and an Andor iXON camera (Andor, Belfast, UK). Images for cell height measurements were obtained using a scanning laser confocal microscope (Olympus IX-81 with an FV-1000 confocal head) with a 30x silicone oil immersion objective (UPLSAPO, Olympus, N.A. $=1.05$, working distance: $0.8 \mathrm{~mm}$ ).

Confocal microscopy for protein localisation

Cells were imaged using either a scanning laser confocal microscope (Olympus IX81 with a FV1000 confocal head or Olympus IX83 with a FV1200 confocal head) or a spinning disc confocal microscope (Yokogawa). Images were taken using a 100× oil immersion objective (UPLSAPO, Olympus, N.A.=1.4, working distance: $0.13 \mathrm{~mm}$ ) and confocal stacks were acquired at $0.3 \mu \mathrm{m}$ intervals in $\mathrm{z}$.

Fluorescence Recovery After Photobleaching

Fluorescence Recovery After Photobleaching (FRAP) experiments in single rounded cells and monolayers were performed using a $100 \times$ oil immersion objective on a scanning 
laser microscope (FV1200, Olympus). The protocol for the FRAP was as described by Fritzsche et al. ${ }^{85}$. Further information is provided in SI.

\section{Chemical treatments}

To deplete monolayers from their ATP stocks, the normal high glucose growth medium was gradually exchanged with PBS and the monolayers were gently washed with PBS to ensure that no high glucose medium remained on the monolayer and in the dish. PBS was then replaced with a solution containing sodium azide $(4 \mathrm{mM})$ and 2deoxyglucose $(10 \mathrm{mM})$ diluted in imaging medium. After 45-60 min incubation at 37C, collagenase type-II was added to the medium to enzymatically remove the collagen. The monolayers were then incubated at room temperature for $1 \mathrm{~h}$. Finally, the medium was exchanged for fresh imaging medium containing sodium azide and 2-deoxyglucose.

45. For inhibition of myosin contractility, monolayers were treated with $50 \mu \mathrm{M}$ Y27632 (Calbiochem). After digestion of collagen, the medium was gradually replaced with the imaging medium. The drugs were then added to the imaging media and the monolayers were incubated for $1 \mathrm{~h}$ at $37 \mathrm{C}$ for all drugs except Y27632, for which we used a 15 minute incubation. Experiments were then performed in presence of the drugs. Control experiments were carried out with the same protocol but in the presence of DMSO alone.

Immunostaining

Cells were incubated with Leibovitz's L15 for $5 \mathrm{~min}$ at room temperature, before being fixed with $4 \%$ PFA diluted in L15 at room temperature for 15 min. After three 10 min washes with PBS, cells were permeabilised with $0.5 \%$ Triton X in PBS for 5 min on ice.

To block nonspecific binding, cells were incubated in $10 \mathrm{mg}^{\mathrm{ml}}-1$ BSA in PBS for 10 min at room temperature and then washed 3 times with BSA/PBS, with each wash lasting 10 minutes. Next, cells were incubated with Phalloidin 647 (Life technologies, A22287, $1: 200$ dilution) for $1 \mathrm{~h}$ at room temperature. Finally, cells were washed three times with BSA/PBS for 10 min each before being mounted in Fluorsave (Merck Millipore) for imaging.

Fitting the second phase of the relaxation with the Standard Linear Solid (SLS) model The second phase of the relaxation curves (defined for $\mathrm{t}>6 \mathrm{~s}$ ) was fitted with the SLS model, which consists of an elastic branch with stiffness $\kappa$ in parallel with a Maxwell branch (Fig S16a). The Maxwell branch consists of a spring of stiffness $\kappa_{M}$ in series with a dashpot of viscosity $\eta$.

Following application of a step strain $\varepsilon_{0}$ at $\mathrm{t}=0 \mathrm{~s}$, the stress in the Maxwell branch will relax as follows:

$$
\sigma(t)=\varepsilon_{0} \kappa_{M} e^{-\frac{\kappa_{M}}{\pi} t}
$$

The characteristic time $\tau_{\mathrm{M}}$ for this relaxation is:

$$
\tau_{\mathrm{M}}=\frac{\eta}{\kappa_{M}}
$$


Experimental data $\sigma_{\text {raw }}(t)$ was fitted as follows: first the residual stress $B$ was subtracted from the raw stress because it represents the stress in the elastic branch and stays constant over time. Next, the stress in the Maxwell branch (i.e. $\sigma_{\text {raw }}(t)-B$ ) was fitted with the stress relaxation function (3), allowing $\kappa_{M}$ and $\eta$ to vary.

Fitting the second phase of the relaxation with the rheological model

The second phase of the relaxation curves (defined for $t>6 \mathrm{~s}$ ) was fitted with the rheological model shown in Fig 5a, which consists of an elastic branch with stiffness $\kappa$ and an active branch. The active branch consists of a spring of stiffness $\kappa_{A}$ subjected to a pre-strain $\varepsilon^{c}$ that can adapt its resting length $L(t)$ to return to $\varepsilon^{c}$ after extension. Thus, in response to an applied strain $\varepsilon_{0}$ that changes the monolayer actual length $l_{m}$ from $l_{0}$ to $l_{1}$, the monolayer stress is $\sigma(t)=\kappa \varepsilon_{0}+\kappa_{A} \varepsilon^{e}(t)$ with $\varepsilon^{e}(t)=\frac{l_{m}-L(t)}{L(t)}$ and $l_{m}=l_{1}$ the actual length of the monolayer imposed by deformation.

In our modelling, we used the following evolution law for the resting length $L(t)$ of the active branch:

$$
\frac{\dot{L}}{L}=\gamma \frac{\left(\varepsilon^{e}(t)-\varepsilon^{c}\right)}{\left|\varepsilon^{e}(0)-\varepsilon^{c}\right|}
$$

where $\gamma$ is the rate of change in resting length. In choosing our empirical evolution function, we reasoned that the material parameters describing the response of the active element $\gamma, \varepsilon^{c}$, and $\kappa_{A}$ should not change when we fit experimental curves for different applied strain because the initial state of the monolayer is the same (Fig S8, S19).

Following application of a step strain at $\mathrm{t}=0 \mathrm{~s}$ that changes the actual length from $l_{m}=$ $l_{0}$ to $l_{m}=l_{1}$, the monolayer resting length $L(t)$ will adapt. Since the monolayers are prestressed and contractile, the value of the resting length before application of the deformation is given by $L\left(0^{-}\right)=l_{0} /\left(1+\varepsilon^{c}\right)$. This provides the initial pre-strain: $\varepsilon^{c}=\left[l_{0}-\right.$ $\left.L\left(0^{-}\right)\right] / L\left(0^{-}\right)$. Using equation (5), the evolution of the resting length is calculated as:

$$
\left.L(t)=\frac{l_{0}}{1+t\left(1+\varepsilon_{0}\right)}-\varepsilon_{0} e^{\frac{\gamma_{t} t}{\varepsilon_{0}}}\right]
$$

Knowing that $\sigma=\kappa_{A} \varepsilon^{e}$, this will lead to stress relaxation in the active branch after application of deformation of the form:

$$
\sigma(t)=\kappa_{A}\left(\frac{\left(1+\varepsilon_{0}\right)\left(1+\varepsilon^{c}\right)}{\left(1+\varepsilon_{0}\right)-\varepsilon_{0} e^{-\frac{\gamma}{\varepsilon_{0}} t}}-1\right)
$$

where $\varepsilon_{0}$ is the applied strain defined as $\varepsilon_{0}=\frac{l_{1}-l_{0}}{l_{0}}$.

The characteristic time $\tau_{\text {model }}$ for this relaxation can be calculated as:

$$
\tau_{\text {model }}=\left(\left.\frac{d \sigma}{d t}\right|_{t=0}\right)^{-1}(\sigma(\infty)-\sigma(0))
$$




$$
\tau_{\text {model }}=\frac{\varepsilon_{0}}{\gamma\left(1+\varepsilon_{0}\right)}
$$

Experimental data $\sigma_{\text {raw }}(t)$ was fitted as follows: first the residual stress $B$ was subtracted from the raw stress because it represents the stress in the elastic branch and stays constant over time. The pre-stress $\sigma_{c}$ was determined in separate measurements because it cannot be determined during stress relaxation experiments (SI, Fig S17a,c). This pre-stress was then added to the stress in the active branch to yield $\sigma_{A}(t)=$ $\sigma_{\text {raw }}(t)-B+\sigma_{c}$. Knowing that the measured pre-stress $\sigma_{c}$ is equal to $\kappa_{A} \cdot \varepsilon^{c}$, we substituted $\varepsilon^{c}$ with $\sigma_{c} / \kappa_{A}$ in equation (7) and $\sigma_{A}(t)$ was fitted with the stress relaxation function (7), allowing $\kappa_{A}$ and $\gamma$ to vary.

\section{Statistical analysis}

All data analysis and curve fitting were conducted using custom-written code in Matlab. For each dataset, outliers were defined as the values that fell outside the range $\left[q_{1}-\right.$ $\left.w \times\left(q_{3}-q_{1}\right), q_{3}+w \times\left(q_{3}-q_{1}\right)\right]$, where $q_{1}$ and $q_{3}$ were the 25th and 75th percentiles of the data and $w$ was 1.5. Outliers were excluded from statistical analysis. The normality of the data was tested using both Lilliefors and Shapiro-Wilk tests in R, which confirmed non-normality of some datasets. Statistical analysis was performed in Matlab, using a two-sided Wilcoxon rank sum test that does not assume normality of the data. Datasets with $p<0.01$ were deemed to be highly significantly different and are denoted by a double asterisk $\left.{ }^{* *}\right)$. Datasets with $p<0.05$ were deemed to be significantly different and are denoted by a single asterisk $\left({ }^{*}\right)$. Changes with $p>0.05$ or where statistical power was less than 0.8 were considered non-significant. For all boxplots, the edges of the box represent the 25th and 75th percentiles of the data, the red line marks the median and the whiskers extend to include the most extreme data points that are not considered to be outliers. Points on each boxplot represent individual monolayers or cells. Each dataset is pooled across experiments performed on at least 3 individual days.

\section{References}

81. Charras, G.T., Hu, C.K., Coughlin, M. \& Mitchison, T.J. Reassembly of contractile actin cortex in cell blebs. J Cell Biol 175, 477-490 (2006).

82. Kajita, M. et al. Filamin acts as a key regulator in epithelial defence against transformed cells. Nature Communications 5, 4428 (2014).

83. Zartman, J., Restrepo, S. \& Basler, K. A high-throughput template for optimizing Drosophila organ culture with response-surface methods. Development 140, 667674 (2013).

84. Mao, Y. et al. Planar polarization of the atypical myosin Dachs orients cell divisions in Drosophila. Genes \& development 25, 131-136 (2011).

85. Fritzsche, M. \& Charras, G. Dissecting protein reaction dynamics in living cells by fluorescence recovery after photobleaching. Nat. Protocols 10, 660-680 (2015). 
a

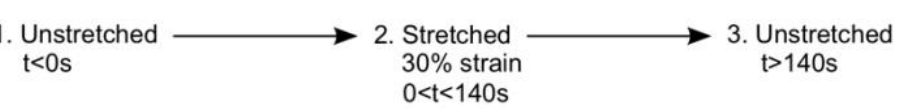

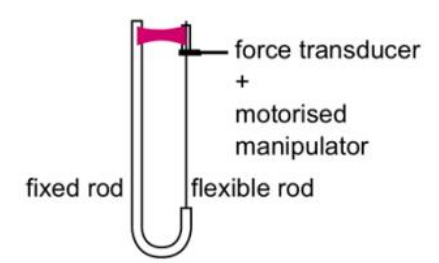

b

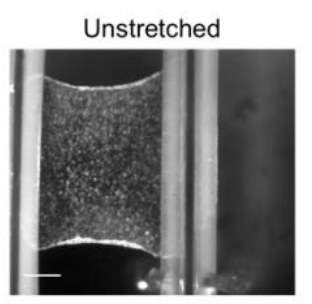

d
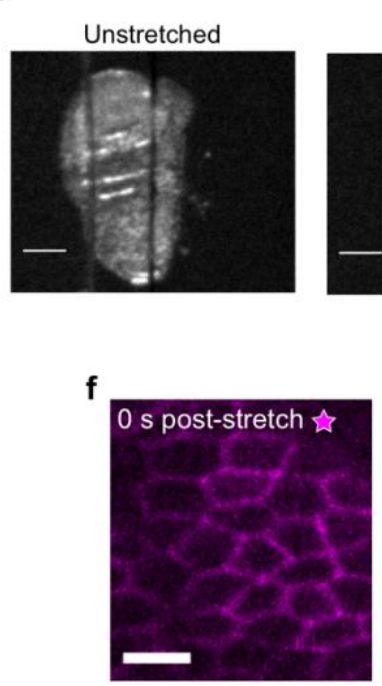

g
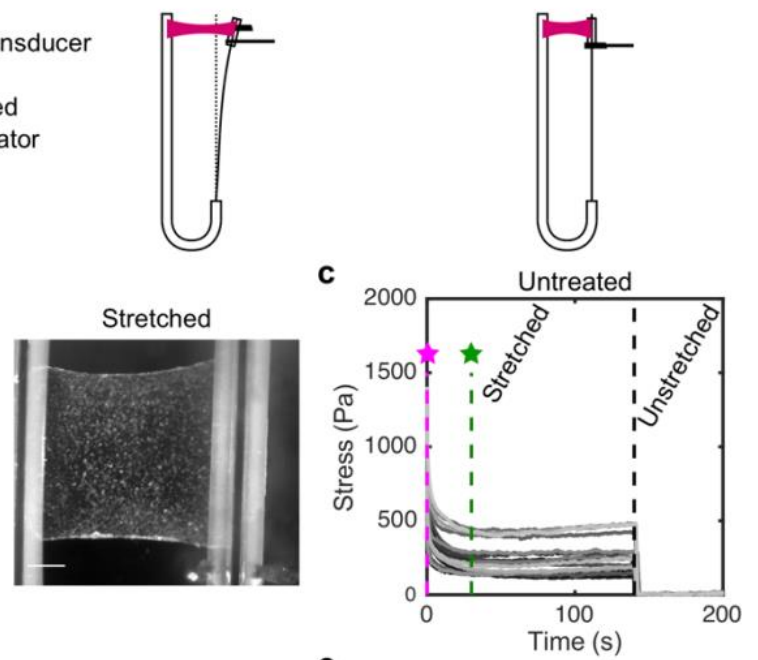

e

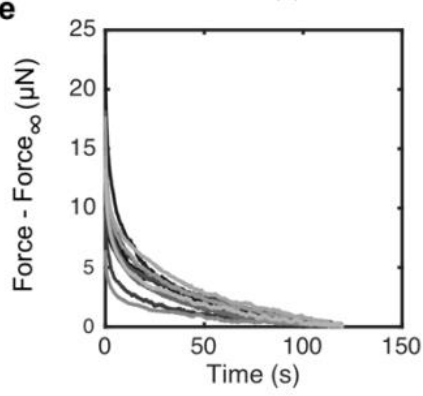

Stretched
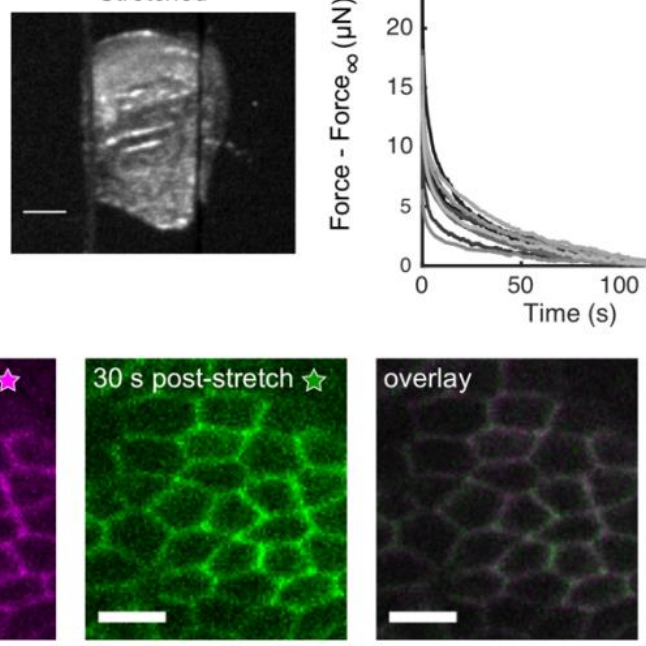

Stretched

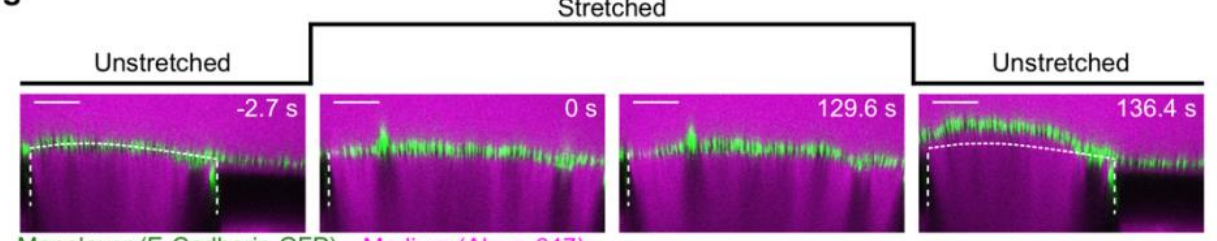

Monolayer (E-Cadherin GFP) Medium (Alexa-647)

Figure 1: Stress relaxation in cell monolayers involves a change in length. (a) Schematic diagram of the stress relaxation experiments. Monolayers were stretched to $30 \%$ strain at a $75 \% . \mathrm{s}^{-1}$ strain rate using a motorised micromanipulator and then kept at a fixed strain for $\sim 130-140 \mathrm{~s}$. The flexible rod was then returned to its initial position and the monolayers were left to recover. (b) Bright-field microscopy images of an epithelial monolayer before and during stretch. (Scale bar: $0.5 \mathrm{~mm}$ ) (c) Stress relaxation curves of cell monolayers $(n=17)$. The magenta and green dashed lines show $0 \mathrm{~s}$ and $30 \mathrm{~s}$ after 
application of stretch. Stresses go to zero upon return of the flexible rod to its initial position ( $\mathrm{t}=140 \mathrm{~s}$, black dashed line). (d) Bright-field microscopy images of Drosophila larval wing discs before and during stretch. (Scale bar: $100 \mu \mathrm{m})(\mathbf{e})$ Stress relaxation curves of Drosophila larval wing discs $(n=12)$. (f) Confocal microscopy images of monolayers expressing E-Cadherin GFP for $0 \mathrm{~s}$ (left) and $30 \mathrm{~s}$ (middle) after stretch. Both images were overlayed to detect potential cell shape change during relaxation (right). $($ Scale bar: $10 \mu \mathrm{m})(\mathbf{g})$ Cross section of a monolayer expressing E-Cadherin GFP before application of stretch $(-2.7 \mathrm{~s})$, during stretch $(0 \mathrm{~s}$ and $129.6 \mathrm{~s})$ and upon release (136.4 $\mathrm{s})$. The length of the monolayer upon release is different from its length before application of stretch. The monolayer appears in green, the surrounding medium appears in magenta due to inclusion of Alexa-647, and the glass substrate appears dark due to dye exclusion. The white dashed lines indicate the positions of the glass substrates. The part of monolayer situated between the two dashed lines is suspended. The dotted white line indicates the shape of the monolayer before application of stretch. (Scale bar: $100 \mu \mathrm{m}$ ) 

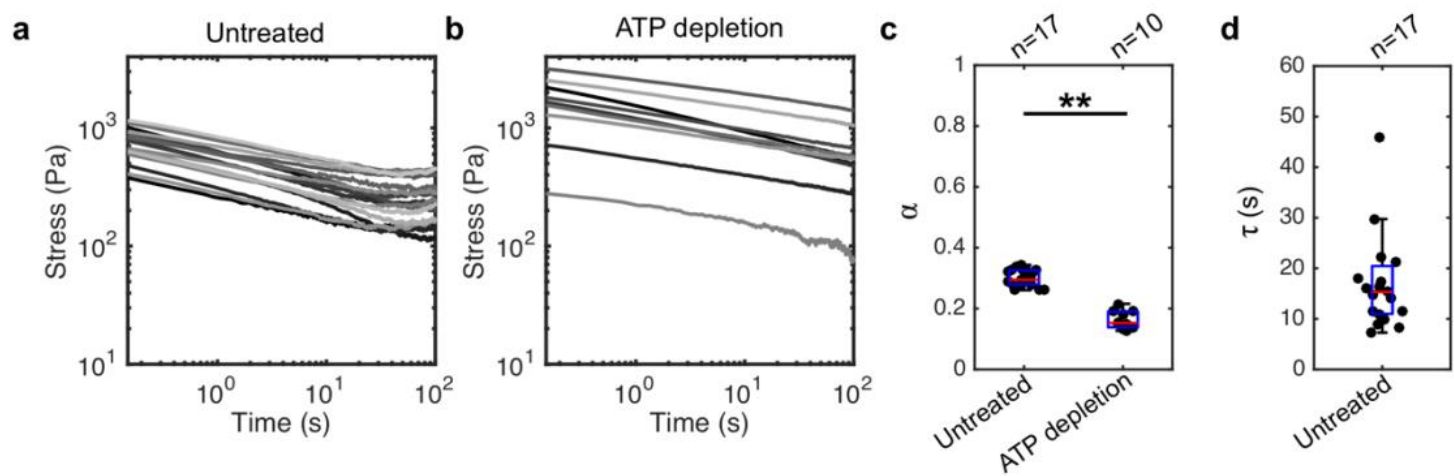

e Actin and Myosin II
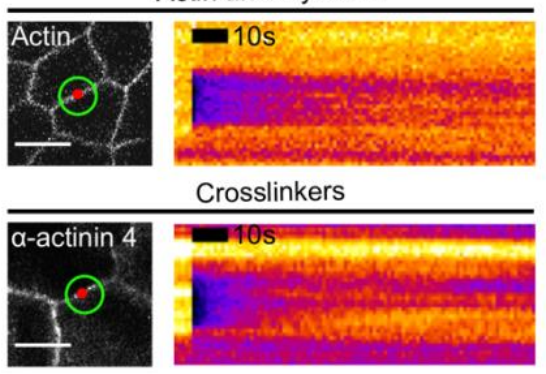

Adherens Junctions

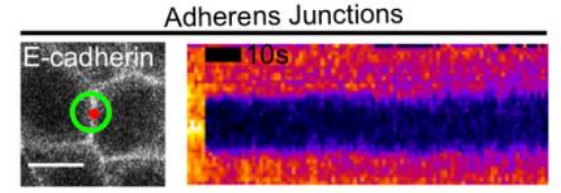

IF and Desmosomes
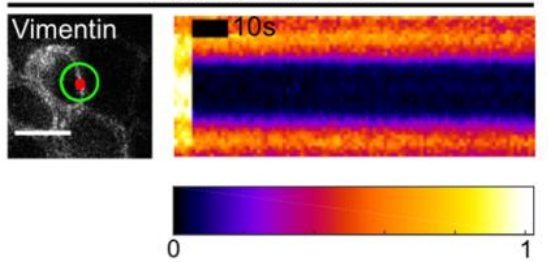

$\mathbf{f}$

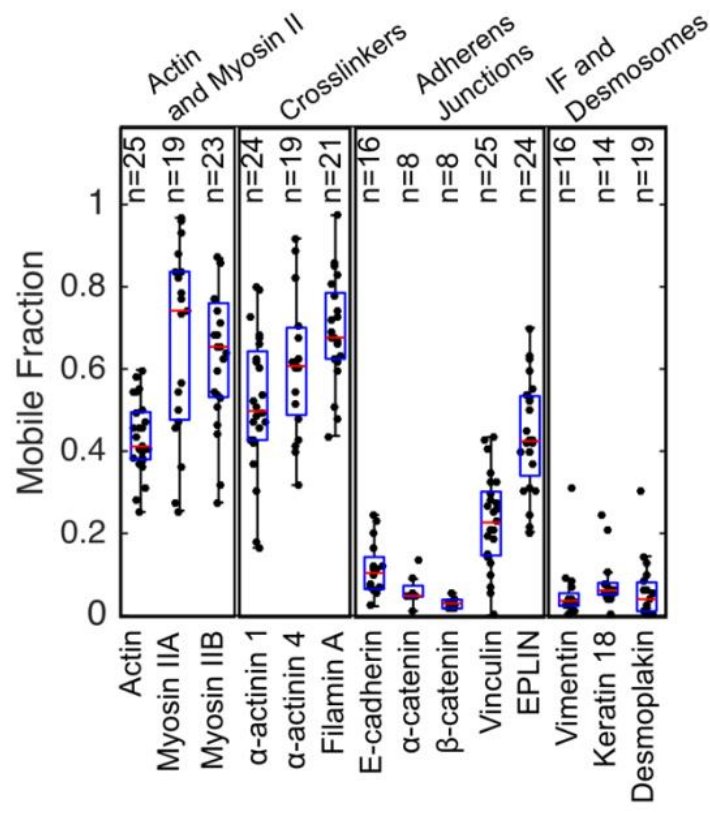

Figure 2: Significant cytoskeletal remodelling occurs over the timescale of stress relaxation. $(\mathbf{a}, \mathbf{b})$ Stress relaxation curves of untreated $(\mathbf{a}, \mathrm{n}=17)$ and ATP depleted $(\mathbf{b}$, $\mathrm{n}=10$ ) monolayers plotted on a logarithmic scale. (c) Boxplots comparing the power law exponent $\alpha$ of untreated and ATP-depleted monolayers. $\left({ }^{* *} p<0.01\right)$ (d) Boxplot of the exponential time constant $\tau$ for untreated monolayers. (e) Confocal microscopy images and kymographs of FRAP experiments. Left panels: the image shows localisation of the protein of interest, the red circle shows the bleached region, and the green circle shows the region imaged for fluorescence recovery. Right panels: each kymograph shows the normalised fluorescence intensity across the junction within the green circle. Intensities are normalised to the maximum intensity in each kymograph. (Scale bar: $10 \mu \mathrm{m})(\mathbf{f})$ Mobile fractions obtained from the FRAP curves for the cytoskeletal, adhesive, and junctional proteins examined. In all boxplots, the number of cells or monolayers examined is indicated above the graph. 
a

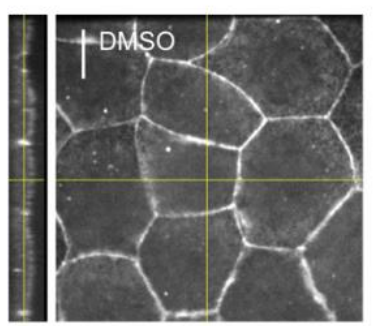

C

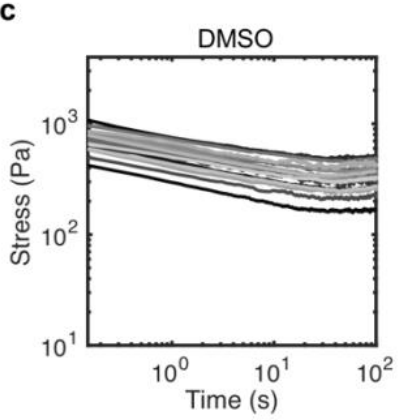

e

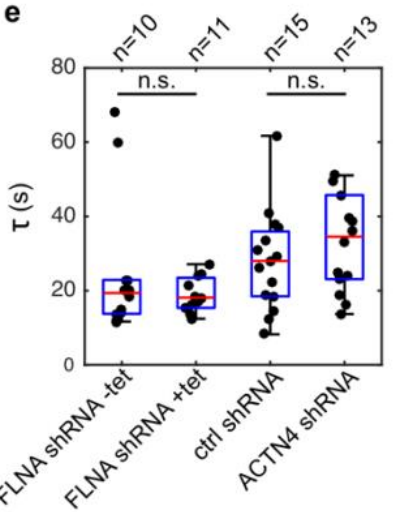

b

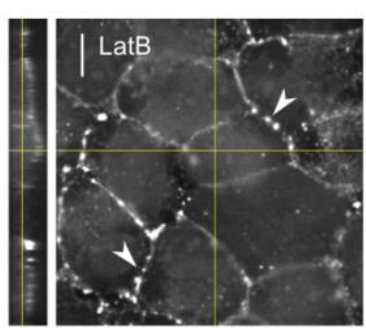

d

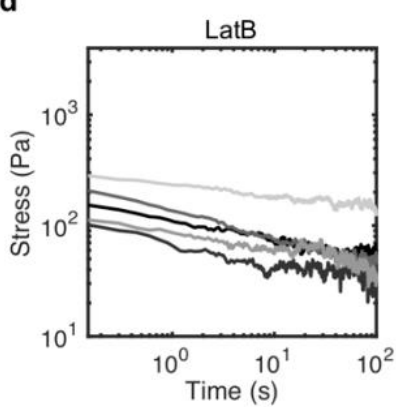

f

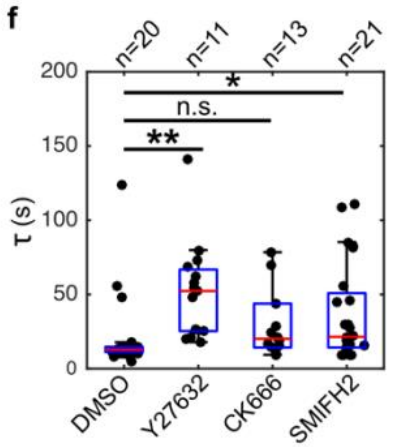

Figure 3: Monolayer stress relaxation is slowed by perturbations to actomyosin. $(\mathbf{a}, \mathbf{b})$ Confocal microscopy images showing F-actin distribution in monolayers treated with DMSO and latrunculin B for $1 \mathrm{~h}$. Junctional actin localisation was perturbed following latrunculin treatment, leaving puncta of actin at the junctions (white arrows). (Scale bar: $10 \mu \mathrm{m})$. The monolayer yz-profile is shown on the left hand side of the xy panel. $(\mathbf{c}, \mathbf{d})$ Stress relaxation curves of monolayers treated with DMSO and latrunculin B for $1 \mathrm{~h}$ displayed in a logarithmic scale. (e) Boxplots comparing the exponential time constant $\tau$ in monolayers depleted for actin crosslinkers Filamin A and $\alpha$-actinin 4 ( $p=0.34$ for FLNA shRNA +tet and $p=0.40$ for ACTN4 shRNA, compared to their respective controls). (f) Boxplots comparing the exponential time constant $\tau$ following treatments with DMSO, Y27632, CK666 and SMIFH2 (** $p<0.01$ for Y27632, n.s. $p<0.05$ with $75 \%$ statistical power for CK666, and ${ }^{*} p<0.05$ for SMIFH2, all compared to DMSO). In all boxplots, the number of monolayers examined is indicated above the graph. 
a

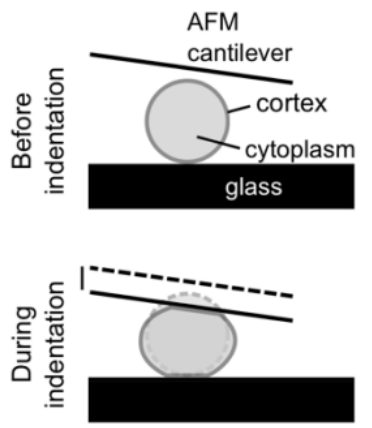

b

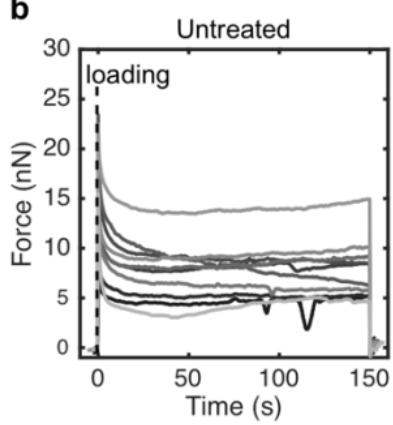

e

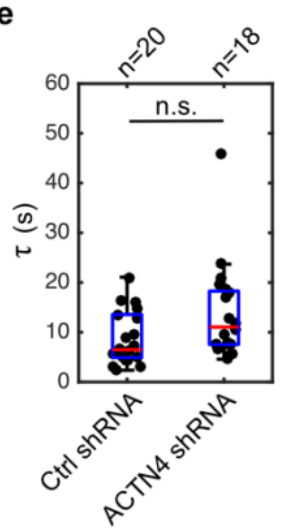

f

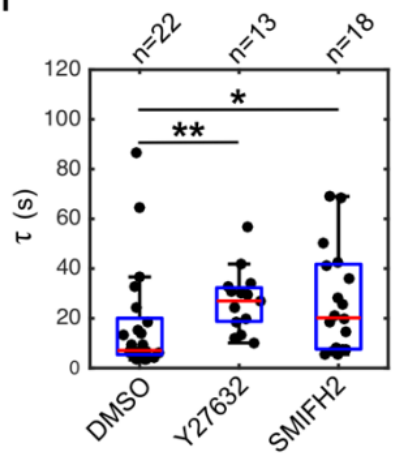

C
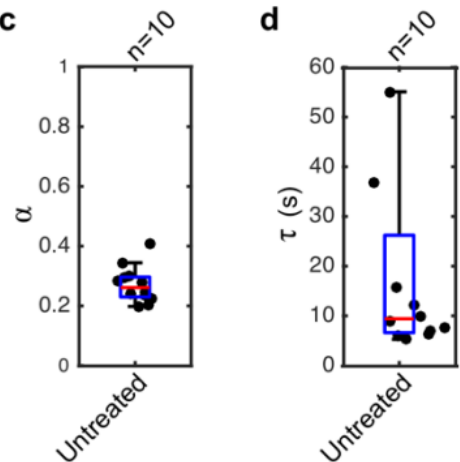

g

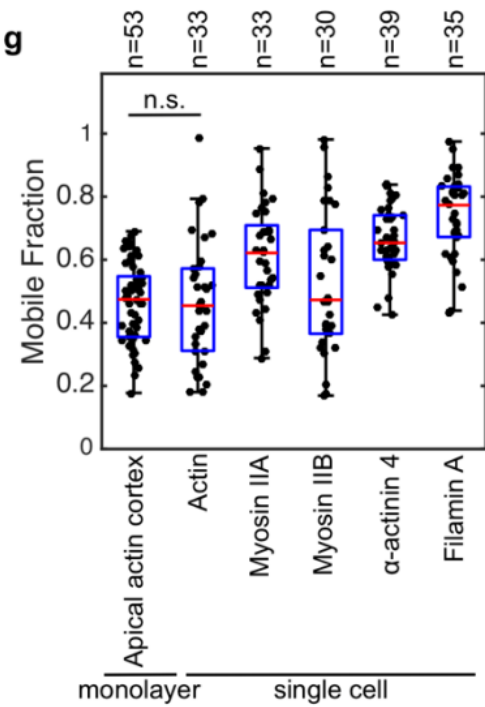

Figure 4: The dynamics of stress relaxation and the extent of actomyosin turnover are similar in single cells and monolayers. (a) Diagram representing the experimental setup. At time $t=0 \mathrm{~s}$, a single rounded cell is compressed between the glass surface and a tipless AFM cantilever. Cell compression leads to stretching of the cortex at the cell free boundaries. The evolution of force over time is measured by monitoring changes in cantilever deflection with an optical lever. (b) Temporal evolution of force in single rounded cells. A step deformation representing $\sim 30 \%$ of cell height was applied at $\mathrm{t}=0 \mathrm{~s}$ and maintained constant for $150 \mathrm{~s}$. Each individual trace corresponds to a different cell. (c-d) Boxplots reporting the power law exponent $\alpha$ and exponential time constant $\tau$ for untreated rounded cells. (e) Boxplots comparing the characteristic relaxation times for cells expressing non-silencing shRNA (Ctrl shRNA) and shRNA targeting $\alpha$-actinin 4 (ACTN4 shRNA) (n.s. $p<0.05$ with $51 \%$ statistical power). (f) Boxplots comparing the characteristic relaxation times for cells treated with DMSO, Y27632, and SMIFH2 ( ${ }^{* *} p<0.01$ for Y27632 and ${ }^{*} p<0.05$ for SMIFH2, both compared to DMSO). (g) Boxplots comparing the mobile fraction of actomyosin proteins in the cortex of rounded cells after $100 \mathrm{~s}$ recovery after photobleaching as well as actin turnover in the apical cortex of cells within monolayers. In all boxplots, the number of cells examined is indicated above the graph. 
a

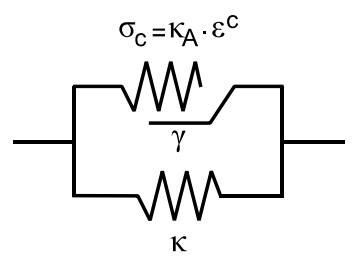

C

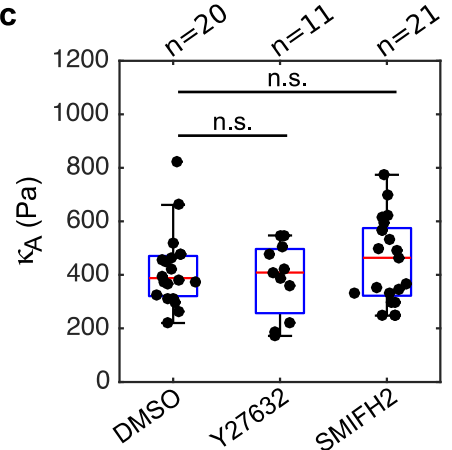

e

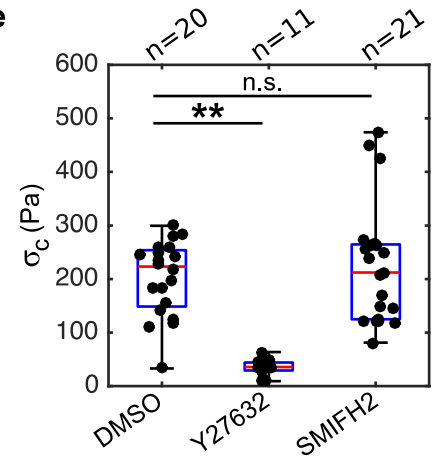

b

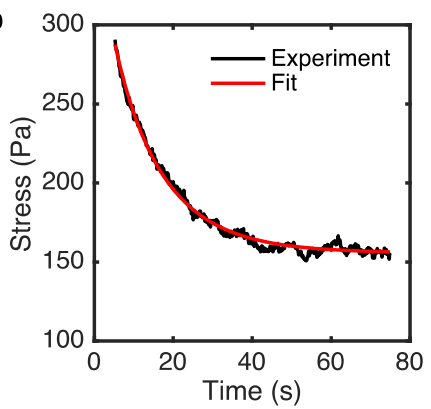

d

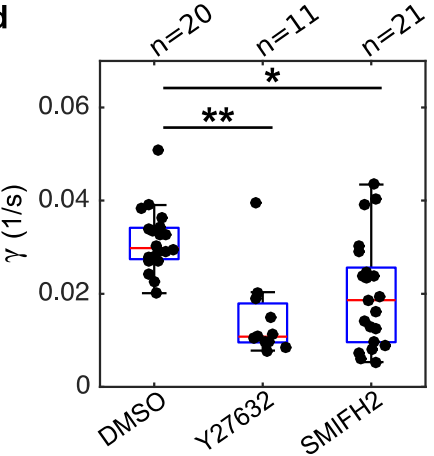

f

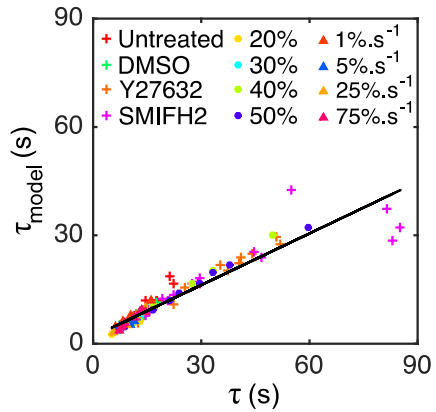

Figure 5: Formin-mediated actin polymerisation and myosin contractility contribute to rheological properties during stress relaxation. (a) Diagram of the rheological model consisting of an active branch (top) and an elastic branch (bottom). The elastic branch consists of a spring with stiffness $\kappa$ and this gives the steady-state behaviour of the monolayer. The active branch describes the transitory regime in response to mechanical perturbation and it comprises an active contractile element that consists of a spring $\kappa_{A}$ subjected to a pre-strain $\varepsilon^{c}$. This active spring can change its resting length $L(t)$ at a rate $\gamma$. (b) The second phase of a representative relaxation curve (black) is fitted with the rheological model shown in a (red). (c,d,e) Boxplots comparing the elastic modulus $\kappa_{A}$, the length-change rate $\gamma$ and the pre-stress $\sigma_{c}$ for monolayers treated with DMSO, Y27632 or SMIFH2. $\left(\kappa_{A}: p=0.95\right.$ for Y27632 and $p=0.58$ for SMIFH2; $\gamma:{ }^{* *} p<0.01$ for Y27632 and ${ }^{*} p<0.05$ for SMIFH2; $\sigma_{c}:{ }^{* *} p<0.01$ for Y27632 and $p=0.80$ for SMIFH2; all compared to DMSO) (f) Time constant $\tau_{\text {model }}$ calculated from the rheological model using equation (9) as a function of the time constant $\tau$ determined from fitting with the empirical function (1) for the different loading regimes and the different perturbations. In all boxplots, the number of monolayers examined is indicated above the graph. 